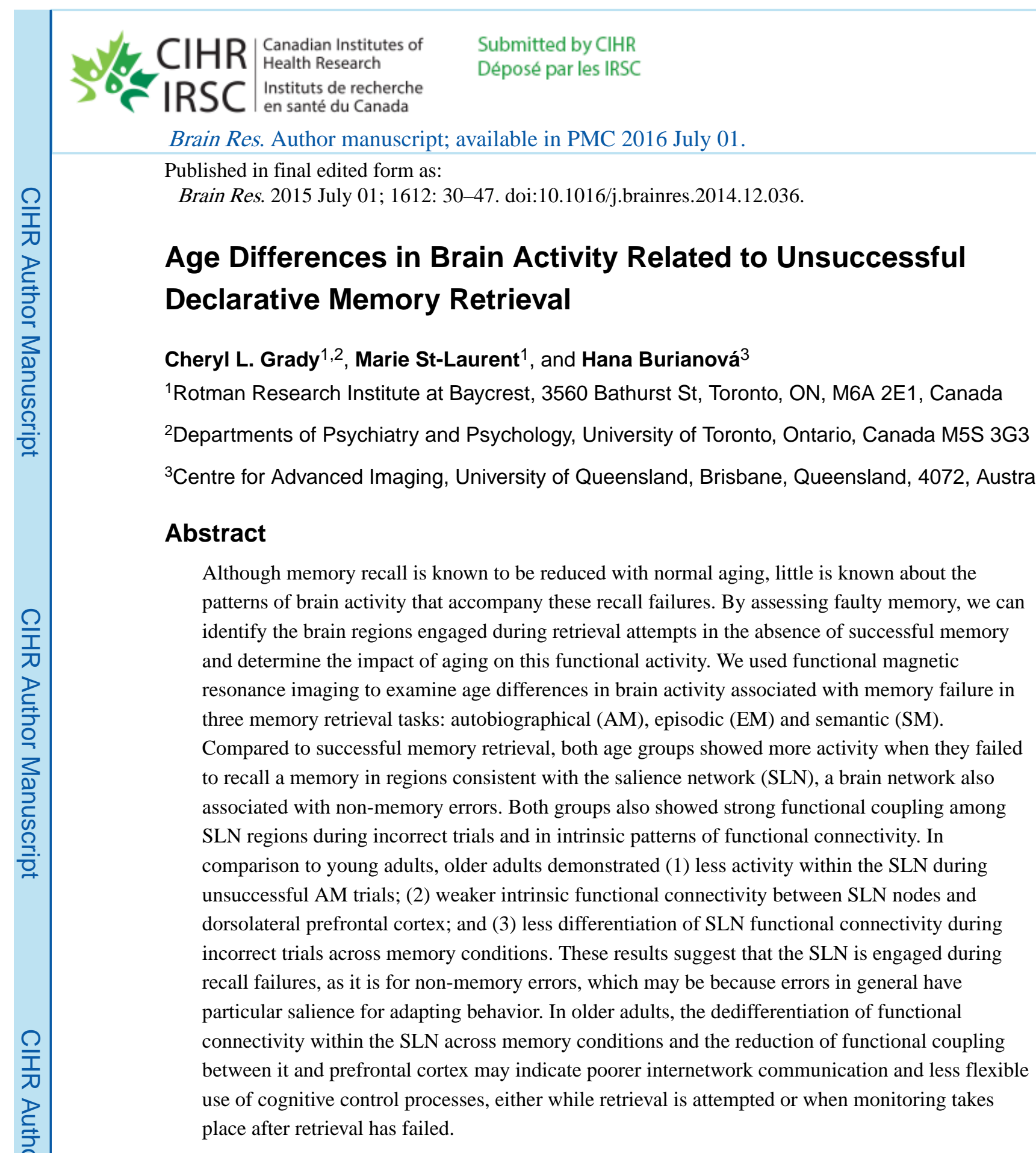

\title{
Keywords
}

fMRI; aging; episodic memory; salience network; frontal lobe

\subsection{Introduction}

A number of studies have provided extensive evidence of the neural circuitry that supports memory retrieval (e.g., Cabeza and Nyberg, 2000; Spaniol et al., 2009; Vilberg and Rugg, 
2008), as well as evidence of how aging influences this circuitry (e.g., Cabeza et al., 2005;

Grady, 2012; Rajah and D'Esposito, 2005). However, most research has investigated how the brain supports successful memory performance (Addis and McAndrews, 2006; Brewer et al., 1998; Morcom et al., 2003; Wagner et al., 1998), and limited attention has been paid to brain networks involved in faulty memory retrieval, most notably faulty long-term memory recall. A number of processes may be engaged during recall, including goal setting, memory search, accessing (hopefully correct) stored memories, and evaluating retrieval success (Henson et al., 1999; Moscovitch, 1992; Rugg et al., 2002). The assessment of faulty memory retrieval can be used to identify brain regions whose activity reflects processes engaged when retrieval fails. Such processes can include cognitive control processes involved regardless of retrieval success (i.e. retrieval attempt), as well as processes related specifically to the failure itself (e.g., error monitoring). In addition, assessing retrieval failure provides an opportunity to explore whether older adults utilize the brain regions involved in unsuccessful memory differently from younger adults.

It is well known that memory failure is common in old age: older adults are prone to make more memory errors, such as false alarms, on episodic memory tasks compared with young adults (Grady et al., 2007; Murphy et al., 2007; Roediger \& Geraci, 2007), and these errors are typically made with higher confidence that the correct answer has been retrieved (Budson et al., 2006; Chua, Schacter, \& Sperling, 2009; Shing et al., 2009). In addition, older adults retrieve autobiographical memories with fewer event-specific details than those reported by younger adults (Addis et al., 2008; Levine et al., 2002; Piefke and Fink, 2005; St Jacques and Levine, 2007). For these reasons, a full understanding of the impact of aging on the neural mechanisms underlying memory requires an assessment of the brain circuits at play when memory fails. With the current study, we aimed to document the neural circuitry engaged when participants failed to retrieve semantic, episodic and autobiographical memories, and to assess whether the engagement of these networks is affected by healthy aging.

As noted above, studies of the neural mechanisms underlying age differences in memory have mostly explored such differences for successful memory processes. For example, a number of experiments have focused on brain activity during encoding of items that are remembered successfully, the so-called subsequent memory effect, and shown differences between younger and older adults indicating that successful encoding is supported by somewhat different brain areas (Cansino et al., 2010; de Chastelaine et al., 2011; Dennis et al., 2007; Duverne et al., 2009; Gutchess et al., 2005; Morcom et al., 2003). Similarly, a number of studies examining brain activity for successful recognition have reported differences between young and older adults in both frontal and parietal cortex (e.g., Daselaar et al., 2006; Duarte et al., 2008; Giovanello and Schacter, 2012; Kalpouzos et al., 2012; Morcom et al., 2007; Rajah et al., 2010; Spaniol and Grady, 2012). A few studies have examined age differences for successful autobiographical memory retrieval (Addis et al., 2011; Maguire and Frith, 2003; St-Laurent et al., 2011) and found differences in medial temporal lobe activity. However, the mechanisms underlying memory errors in older adults have not been explored extensively, although given the increase in memory failures observed in older adults it is important to study the failures as well as the successes. In the following sections, we review evidence for error-related brain activity in young and older adults. 


\subsection{Error-related Brain Activity}

Studies examining the neural processes related to errors on a variety of cognitive tasks in young adults typically show increased activity in both medial and lateral prefrontal cortices (PFC). For example, electrophysiological studies of speeded response tasks have shown increased activity in medial frontal regions, localized to the anterior cingulate cortex (ACC), and attributed this activity to error monitoring processes necessary for potential modification of performance (for reviews see Holroyd and Coles, 2002; van Veen and Carter, 2006). Functional MRI studies have found more activity in the ACC and a region at the intersection of the inferior frontal operculum and anterior insula (aIFO) for errors than for correct responses during a variety of tasks, including visual search and semantic judgments (Dosenbach et al., 2006), anti-saccade tasks (Klein et al., 2007), Simon tasks (Ham et al., 2013b) and stop signal tasks (Rubia et al., 2007). This prominence of error-related activity in aIFO and ACC suggests the engagement of a brain network known as the salience network (SLN). This network includes these areas, as well as the supramarginal gyri, ventral striatum and amygdala, and is thought to be driven by the salience or importance of stimuli in the environment (Downar et al., 2002; Seeley et al., 2007). Indeed, at least one study has directly attributed error-related activity during a Simon task to the SLN (Ham et al., 2013a). Thus, committing errors may be a particularly salient event that provides individuals with information that can be used to modify behavior accordingly.

In memory experiments carried out in younger adults, error-related activity has been found in SLN regions such as the ACC and supramarginal gyrus during false recognition (Slotnick and Schacter, 2004) and reality monitoring tasks (Okado and Stark, 2003), and in ACC and aIFO during missed source judgments and false alarms, relative to correct source judgments (Donaldson et al., 2009). Error-related activity also has been reported for false alarms vs. hits in ventromedial PFC (Cabeza et al., 2001), and left temporal cortex (Garoff-Eaton et al., 2006). However, in recognition paradigms it is not clear if participants are even aware of their errors, whereas in other kinds of tasks this is either assumed or assessed explicitly (Klein et al., 2007; Ham et al., 2013). A memory paradigm used by Maril and colleagues (Maril et al., 2001; Maril et al., 2005) assessed retrieval failures accompanied by awareness on the part of the participant by assessing the tip-of-the-tongue (TOT) phenomenon. In TOT, participants are required to indicate when they cannot retrieve the required information but feel that this retrieval is imminent. In these studies (Maril et al., 2001; Maril et al., 2005), both successful recall and TOT events were accompanied by increased activity in ACC and aIFO, but the increases were larger and more sustained during TOT, indicating that memory retrieval failure accompanied by awareness also involves SLN regions.

\subsection{Errors and Aging}

To-date, aging studies have found that recognition failures in older adults are associated with more activity in brain regions that process irrelevant stimuli during encoding (Stevens et al., 2008). False alarms during recognition memory tasks, relative to hits or correct rejections, have been related to greater activity in left temporal cortex (Dennis et al., 2008) and hippocampus (Giovanello et al., 2010) in older relative to younger adults, and to less activity in precuneus (Duarte et al., 2010). In contrast, electrophysiological studies of non-memory errors generally have found smaller error-related responses linked to the ACC in older 
compared to young adults (Band and Kok, 2000; Endrass et al., 2012; Falkenstein et al., 2001; Nieuwenhuis et al., 2002). Thus, the literature on brain activity during errors in older adults is not extensive, and does not point to the SLN specifically, although some work does indicate age differences in the ACC.

\subsection{The Current Study}

We examined brain activity during declarative memory retrieval failures in young and older adults to determine whether these errors are accompanied by heightened activity in the SLN, and whether such activity differs with age. Given the studies showing SLN activity for memory errors, including TOT, we reasoned that the failure to recall a specifically-cued memory also should engage the SLN, presumably reflecting both error processing and response monitoring (Ham et al., 2013a). Cued recall errors would be similar to TOT or other types of conscious errors because individuals would be aware that they have only partially succeeded or not succeeded in retrieving a memory related to the cue. Thus, the purpose of the current study was to examine how age affects the neural circuitry engaged when participants attempt but fail to recall long-term memories.

Using fMRI data from a previously published study (St-Laurent et al., 2011), we examined brain activity from young and older adults during incorrect vs. correct memory decisions across three conditions requiring retrieval of different memory content: Autobiographical (AM—personal life events), Semantic (SM—general knowledge) and Episodic (EM—events encoded in the laboratory). In this study, participants were cued to retrieve one of these three types of memory. If an AM was cued, they judged whether the retrieved memory was either very vividly or somewhat vividly retrieved, or whether no relevant memory could be recalled. For the other two conditions, participants either answered a general knowledge question (SM), or a question related to a picture shown earlier in the experiment (EM), and the response could either be correct or incorrect, or participants could indicate that they did not know the answer. Our focus in the current study was on brain activity during unsuccessful trials, defined as trials with incorrect and "I don't know" responses for EM and SM. For AM, incorrect trials were those given "somewhat vivid" or "I don't know" responses. We assessed activity across incorrect and correct trials using a multivariate analysis to determine if there was a distributed pattern of brain activity, consistent with the SLN, that would be more active during incorrect than correct trials across all memory conditions. In both groups of participants, we expected to see greater activity in areas such as ACC and aIFO for incorrect responses and failures to retrieve a memory, consistent with the literature cited above. Given the age reductions in brain activity observed for AM and EM relative to SM during successful trials (St-Laurent et al., 2011), we expected age differences in activity during unsuccessful trials to emerge primarily in the two "episodic" conditions.

To address whether the entire SLN was active for memory failures, we also examined functional connectivity within this network, defined using two major nodes of the network (see section 2.4), during incorrect trials and assessed age differences in this functional coupling. Age differences in functional connectivity have been examined extensively for the default network (e.g., Andrews-Hanna et al., 2007; Damoiseaux et al., 2008; Grady et al., 
2012), and to some extent for other brain networks (Allen et al., 2011; Campbell et al., 2012; Onoda et al., 2012; Rieckmann et al., 2011; Thomas et al., 2013; Tomasi and Volkow, 2012; Voss et al., 2010). Weaker functional connectivity has been reported specifically within the SLN or in SLN nodes in older adults (Allen et al., 2011; Geerligs et al., in press; He et al., 2014; Meier et al., 2012; Onoda et al., 2012; Tomasi and Volkow, 2012), leading us to predict age-related reductions in SLN functional connectivity in our results, although connectivity within the striatum, a node of the SLN, has been shown to increase with age (Allen et al., 2011; Tomasi and Volkow, 2012; Wang et al., 2012). However, most of these earlier studies examined resting-state functional connectivity, and it is unclear whether similar age reductions would be found during our tasks, on which there were no age differences in overall accuracy or response times (St-Laurent et al., 2011). Previous work also has shown age-related reductions in connectivity between areas from the frontoparietal control network, or FPC (Spreng et al., 2013; Vincent et al., 2008) and SLN areas, such as the aIFO (Campbell et al., 2012; He et al., 2013). Thus, we anticipated that age differences might emerge in the strength of functional connections between SLN nodes and other brain regions, particularly those involved in cognitive control.

\subsection{Results}

\subsection{Behavior}

We previously reported that there were no age differences in the proportion of trials in which participants were able to retrieve a very vivid AM or a correct EM or SM (St-Laurent et al., 2011). For the purposes of the present report, reaction time (RT) data were analyzed with a two (group) $\mathrm{x}$ three (memory task) $\mathrm{x}$ two (correct, incorrect) ANOVA. There were significant main effects of task, $F(2,56)=19.2, p<0.001$, and type of response, $F(1,28)=$ 128.9, $p<0.001$. Incorrect RTs were significantly slower than correct ones (Figure 1), and RTs for AM and SM were significantly slower than for the EM condition (both $p s<0.001$, corrected). We observed no significant effect of age group, and no interactions were significant.

\subsection{Brain Activity during Incorrect Trials}

Image analysis was conducted with a multivariate approach (partial least squares, or PLS), which identifies spatiotemporal patterns of whole-brain activity that covary with task effects (Krishnan et al., 2011; McIntosh et al., 1996; McIntosh and Lobaugh, 2004). Using this data-driven approach to assess patterns of activity during correct and incorrect trials, we identified a set of regions that distinguished unsuccessful from successful trials across conditions ( $p<0.004$; the $p$ value here applies to the entire pattern of activity across the brain, see Figure 2a). To assess the magnitude of brain activity in these regions as a function of condition, we examined the summary measures that indicate the extent to which each participant expresses the whole-brain activity pattern seen in Figure 2a (known as "brain scores") over the time points in the $16 \mathrm{~s}$ analysis window. We first tested the hypothesis that there was more activity overall for incorrect than correct trials, by collapsing across memory condition and group and using a repeated measures ANOVA with response and time as factors. The effect of response was significant $(F(1,29)=29.36, p<0.001)$, as was the effect of time $(F(6,174)=10.58, p<0.001)$, and the response $\mathrm{x}$ time interaction $(F(6,174)=13.3$, 
$p<0.001)$. This indicates that there was more activity for incorrect than for correct responses within the regions identified by the PLS pattern, and that this difference increased over time (Figure $2 \mathrm{~b}$ ). In addition, the whole-brain response (averaged across time) was significantly different from zero for incorrect trials (mean $\pm \mathrm{SE}, 2.46 \pm 0.37$; one sample ttest, $t(29)=6.55, p<0.001$, corrected for 2 comparisons), but not for correct trials (mean, $0.01 \pm 0.25 ; t<1$ ). That is, activity in the regions seen in Figure 2a, as a group, was increased only during incorrect trials.

More activity for incorrect responses was seen in aIFO bilaterally, ACC, thalamus, caudate, supramarginal gyrus (SMG) and midbrain (Figure 2a, Table 1), all of which are thought to be nodes of the SLN (Downar et al., 2002; Seeley et al., 2007). Additional regions with more activity for incorrect trials included frontal areas associated with the frontoparietal control network, or FPC (Vincent et al., 2008), such as the middle frontal gyrus. We note that there was some overlap between the regions identified in Figure $2 \mathrm{a}$ (incorrect > correct), and regions that were found in our prior work to be more active during the same three memory conditions compared to a non-memory control task (Burianova and Grady, 2007; St-Laurent et al., 2011). As shown in Supplementary Figure 1, some SLN regions showed more activity across the memory conditions compared to the control task (e.g., aIFO), but other areas that showed more activity for unsuccessful than for successful trials did not differ between the memory conditions and the control task (e.g., right supramarginal gyrus). Therefore, some, but not all SLN regions were active during memory retrieval in general, while all showed a heightened engagement during recall failure.

Having identified a significant whole-brain response in the SLN that was specific to incorrect trials, we then tested for condition and group effects on the brain scores from the incorrect trials using an ANOVA with group, condition, and time as factors. The main effect of condition was not significant, but the main effect of time $(F(6,168)=16.97, p<0.001)$ and the interaction of group $\mathrm{x}$ condition $(F(2,56)=8.66, p=0.001)$ were significant (see Figure 3). This interaction remained significant when the ANOVA was carried out using mean activity for the correct trials as a covariate $(F(2,54)=3.91, p=0.03)$, indicating that the differential engagement of the SLN as a function of group and condition was specific to unsuccessful trials. During unsuccessful AM trials, the young adults had significantly greater SLN activity than the older adults $(F(1,28)=7.95, p<0.01)$. In contrast, there was a trend for greater activity in older adults during incorrect EM trials $(F(1,28)=3.59, p=0.07)$ and no group difference for SM trials $(F<1)$. In other words, young adults were more likely to engage SLN regions during the unsuccessful retrieval of personal memories (AM), whereas older adults tended to have more of this activity for non-personal memories (EM).

It is important to note that these patterns of brain activity were observed in the context of RT differences between correct and incorrect responses (Incorrect > Correct, Figure 1). The nature of our tasks makes it likely that unsuccessful memory retrieval resulted in the engagement of additional cognitive processes that contributed to longer response times. Thus, it is difficult to fully separate the influence of these processes and RT on brain activity. Nevertheless, we considered that some assessment of the strength of the influence of RT on activity during incorrect trials was warranted. First, we assessed the influence of RT on activity during incorrect trials using the brain scores (averaged over time) as a measure of 
this activity, because these scores reflect how much each participant expresses the pattern of activity seen in Figure 2a. We used an ANCOVA to assess group effects on brain scores for incorrect trials in the three conditions (AM, EM and SM), with mean RTs from each condition entered as covariates. In this analysis, only the group $\mathrm{x}$ condition interaction was significant, $F(2,50)=6.3, p=0.004$. A similar result was found when the differences in RT between correct and incorrect responses for each condition were used as the covariates: group $\mathrm{x}$ condition interaction, $F(2,50)=8.0, p=0.001$. These analyses indicate that even after accounting for the influence of RT on brain activity, the age differences apparent in Figure $2 \mathrm{~b}$ were still robust. In addition, correlations were calculated between participants' brain scores from incorrect trials and their mean RT for these trials in each condition; these analyses were conducted across young and old participants combined, as there were no group differences in RT. The only significant correlation between brain activity and RTs was for the incorrect EM trials $(r=0.44, p=0.015)$. These additional analyses indicate that, in general, the error-related brain activity that we observed was not strongly influenced by RT.

\subsection{Response profiles in alFO}

To focus on the SLN for the functional connectivity analyses described below, we chose two regions, the right and left aIFO, as "seeds" and used PLS to measure the correlation between activity in these seeds and the rest of the brain during incorrect trials. The aIFO was chosen because it is considered a major SLN node and has been used by others to define this network (Farb et al., 2013; Seeley et al., 2007). To illustrate activity in these seed regions, Figure 4 shows the hemodynamic response of the right and left aIFO for young and older adults during correct and incorrect responses, averaged across the three memory conditions. There are two things to note about this activity. First, it appeared to increase for both correct and incorrect trials, unlike the whole-brain response shown in Figure 2, which increased reliably only for incorrect trials. Figure 4 also shows the response of another SLN region, the right SMG, during incorrect and correct trials; activity in this region appeared to increase only near the end of incorrect trials. A similar response profile was seen in the right superior frontal gyrus, one of the PFC regions that were more active for incorrect responses, along with the SLN. Activity in this region appeared to briefly decrease and then increase during incorrect trials. These profiles indicate that the individual responses to task demand are variable among SLN regions; nevertheless all of the response profiles are consistent with the whole-brain profile of increasing difference between correct and incorrect response over time.

The second thing to note about activity in the aIFO is that it appeared to be sustained longer during incorrect trials. To determine whether the difference in activity between incorrect and correct trials was most robust late in the trial, we carried out ANOVAs with trial type (correct, incorrect) and time (time points 2-8) as repeated measures for the right and left aIFO. For the right aIFO, the critical interaction of trial type and time was significant, $F(6,168)=10.5, p<0.005$ (there were no significant effects involving group). Activity for incorrect trials was higher than activity for correct trials in the last two time points of the trial ( $p<0.05$, corrected for multiple comparisons). Similarly, for the left aIFO, the interaction of trial type and time was significant, $F(6,168)=13.0, p<0.001$ (there were no significant effects of group), and activity was higher for incorrect than for correct trials in 
the last two time points of the trial ( $p<0.05$, corrected for multiple comparisons). In sum, in both seed regions, the response was more sustained during trials in which participants could not retrieve the cued memory, consistent with the greater difference over time between incorrect and correct trials seen in the whole-brain response (Figure 2b).

\subsection{Task-Related Functional Connectivity}

We next examined whether the regions active for incorrect responses were functionally connected, i.e., could be considered a network, and whether age modulated their pattern of functional connectivity. Using the right/left aIFO regions as seeds, we assessed functional connectivity patterns during the incorrect trials across the three memory conditions. The first significant pattern of functional connectivity revealed by PLS $(p<.005)$ identified regions with strong functional coupling to the aIFO seeds across conditions and groups, including SLN areas such as the ACC, striatum, supramarginal gyrus and midbrain (Table 3 and Figure 5a). Other regions with strong connectivity to the seeds included frontoparietal cognitivecontrol regions, such as middle frontal gyri and inferior parietal lobes. Overall, these regions closely resembled those with increased activity for incorrect trials, although the spatial pattern was more extensive (compare Figure 2a and 5a). Correlations between brain scores -which reflected to what extent a participant's brain activity resembled the spatially distributed pattern shown in Figure 5-and activity in each seed are reported per condition and age group in Table 2; for this pattern, no correlation differed significantly between the two age groups.

A second significant pattern of functional connectivity $(p<0.02)$ showed a clear distinction between AM and SM/EM in young adults (warm colored regions in Figure 5b, Table 4). The aIFO seeds correlated positively with vmPFC and the superior frontal gyrus, angular gyrus, amygdala/parahippocampal gyrus and striatum in the left hemisphere during AM (Table 4); during EM and SM positive correlations were seen in bilateral precentral gyri, and some temporo-occipital regions (cool colored regions in Figure 5b, Table 4). In contrast, older adults showed no differences in the correlation values across the memory conditions in these regions (Figure 5b), and most correlations between brain scores and seed activity were not reliably different from zero (Table 2). In addition, age differences were seen for connectivity with the right aIFO seed in AM and for connectivity with both seeds in SM (Table 2).

To determine if this second pattern of functional connectivity was specific to the incorrect trials, we carried out an analysis of the correct trials using the same two seeds in aIFO. Unlike for incorrect trials, for correct trials there were no significant patterns of functional connectivity that differentiated the memory conditions in young adults, but not older adults (as seen for incorrect trials in Figure 5b). Instead, there was a limited set of regions with non-specific age differences across all three memory types (see Supplementary Figure 2). In addition, there were no significant correlations in either age group (all $p s>0.05$, corrected for 6 comparisons) between the brain summary scores from the pattern of connectivity for incorrect trials (Figure 4b) and those for correct trials (Supplementary Figure 2, Latent Variable 2), suggesting that the pattern seen in Figure $4 \mathrm{~b}$ was specific to the incorrect trials. 


\subsection{Intrinsic Functional Connectivity}

In addition to examining SLN functional connectivity during task, we considered it important to obtain an estimate of this network's intrinsic functional connectivity in each age group. Intrinsic functional connectivity is thought to reflect the spontaneous communication among brain regions that exists when a person is at rest (Boly et al., 2008; Fox et al., 2005; Mennes et al., 2013), but nevertheless reflects the range of functional connections that are available during task performance (Smith et al., 2009). Importantly, intrinsic functional connectivity is related to cognitive performance in both young and older adults (Andrews-Hanna et al., 2007; Dang et al., 2013; Kelly et al., 2008). To examine intrinsic functional connectivity, we analyzed activity correlated with the aIFO seeds across a functional run after removing the effect of the task events. This type of approach has been used by others (Al-Aidroos et al., 2012) and shown to result in patterns of functional connectivity that closely resemble those calculated from "true" resting state scans (Grady et al., 2014; Grigg and Grady, 2010a).

The functional connectivity pattern accounting for the most covariance in the data is shown in Figure $6 \mathrm{a}(p<0.001)$. The spatial pattern of brain regions was similar to the set of regions with increased activity for errors (Figure 2a) and to the common task-related functional connectivity pattern (Figure 5a); indeed, there was considerable overlap among these three spatial patterns (Figure 7). The bilateral aIFO seeds were functionally coupled to ACC, striatum, thalamus, and middle frontal cortex bilaterally (Table 3). Robust correlations between seed activity and activity in the regions shown in Figure 6a were seen in both age groups. A two (group) by two (hemisphere) ANOVA conducted on the correlations (after converting them to $\mathrm{Z}$ scores using the Fisher r-to- $\mathrm{Z}$ transformation) showed that neither the group effect $(F[1,78]=3.3, p=0.07)$ nor the group $\mathrm{x}$ hemisphere interaction $(F[1,78]=2.3$, $p=0.13)$ was significant.

A second significant functional connectivity pattern also was identified (Figure 6b, $p<$ $0.001)^{1}$. In this pattern, the aIFO seeds were correlated with bilateral middle frontal cortex and dorsomedial PFC, plus a left temporal region (Table 4) in both groups, but these correlations were weaker in the older adults compared to the younger adults $(F 1,78]=4.0$, $p<0.05$; Figure $6 \mathrm{~b}$ ). A few regions showed negative correlations with the seeds, including precuneus $(4,-76,36, B S R=6.2)$ and mid-cingulate $(0,-4,48, B S R=-5.8)$.

Although we attempted to remove the influence of task on these functional connectivity results, it is possible that some of the similarity between the functional connectivity patterns we observed across the task and intrinsic functional connectivity analyses (i.e., the similarity between the regions seen in Figures 5a and 6a) could be due to the fact that the latter assessment was done on a task run and not on "true" resting state scans. To examine this issue, we carried out a functional connectivity analysis, using the same two seed regions, in an independent group of young adults who were scanned at rest ( $\mathrm{n}=45 ; 18-29 \mathrm{yrs}, M=$ 22.4, $S D=3.1 ; 23$ males). The "true" resting pattern of functional connectivity within the SLN is shown in Supplementary Figure 3, and was very similar to the task-related and

${ }^{1}$ Note that additional significant Latent Variables were identified in this analysis, but because they each accounted for less than $2 \%$ of the total covariance, they are not reported here. 
intrinsic patterns, showing considerable overlap with them. Thus, the network functional connectivity seen here is not due to our particular dataset or to the way we removed the effect of the task events, but instead highlights the robustness of the correlations with the aIFO seeds across multiple analyses and experimental conditions.

\subsection{Discussion}

In this experiment, we compared patterns of brain activation elicited during memory retrieval errors in young and older adults, using a cued recall task, and addressed two main questions: 1) is the SLN involved in memory recall errors, and 2) does activity or functional connectivity differ with age during recall failures? In both young and old adults, we found that unsuccessful retrieval was associated with more activity than successful retrieval in a widespread set of regions that have been previously associated with monitoring, stimulus salience, and error processing. This activity was seen primarily in regions thought to be nodes of the SLN, although frontal control regions also were active. These results suggest that recall failures may be especially salient events that are associated with more SLN activity than successfully recalled memories. In terms of age differences, younger adults showed more activity than older adults during trials for which they were unable to retrieve a vivid personal memory (AM). Strong intrinsic functional connectivity and functional connectivity during the memory tasks was observed within the SLN in both young and older adults. Despite maintained functional connectivity within the SLN, however, older adults had less distinct patterns of connectivity across the memory conditions, relative to young adults, and weaker intrinsic connectivity between the SLN and dorsolateral PFC. Overall, these results indicate that age differences in how young and older adults recruit the SLN when unsuccessfully attempting to retrieve declarative memories vary as a function of the type of memory content to be retrieved.

\subsection{Greater Activity for Incorrect vs. Correct Trials}

As expected, we found that when people are unable to retrieve correct information or a vivid personal memory, they engage the SLN, as well as other PFC areas involved in cognitive control. The summary measure of activity in these regions was significantly increased only for incorrect trials, and difference in activity between incorrect and correct trials increased within a trial's time window. It is not possible from this study to ascertain whether activity during incorrect trials reflects some particular process occurring at the time of the error, the evaluation of the error after the participant has failed to retrieve the cued memory, or a longer engagement of the same processes that also occur when memory succeeds. The differing response profiles of some SLN regions, and the fact that only some of them are active across memory conditions vs. a control task, suggest that SLN nodes may play different roles in memory retrieval. That is, some SLN regions (e.g., aIFO) may be active for retrieval regardless of the outcome, probably for monitoring and cognitive control, and continue to play this role longer when retrieval is not successful. Activity in other regions (e.g., SMG) may underlie error-specific processing or post-error evaluation. However, since our experiment was not designed to disentangle these different processes, we conclude that at least some of what we have observed in this study likely reflects types of processing that can occur regardless of retrieval success, with heightened and/or prolonged processing when 
retrieval fails. Clearly, experimental work addressing this question of processing specificity during errors needs to be done in the future. Regardless, our results show that the SLN responds to a greater degree for failed recall attempts than for successful ones, a difference that increases with time as the contribution of each region comes online.

Our interpretation that at least some SLN regions are engaged during both correct and incorrect trial types, but this activity is sustained longer for incorrect trials, is consistent with what others have reported for the aIFO (Maril et al., 2005; Wheeler et al., 2008). The pattern of activity that we observed in aIFO is also consistent with previous work (Carter et al., 1998) showing that some "error-related" activity also occurs during correct trials in which there is high response competition, which may have been a factor in our study (i.e., competition among details that may or may not be pertinent to the recalled memory). Thus, our results, taken together with the error literature in general, support the idea that heightened and/or sustained SLN activity occurs for recall failures compared to retrieval successes, similar to that seen for non-memory errors, although individual SLN nodes may have different response profiles. Heightened engagement of the SLN may be a general phenomenon that does not depend on the particular context in which the error occurs, but may indicate high salience of the event due to its potential impact on future behavior.

We originally hypothesized that age differences in error-related activity would be greater in the AM and EM conditions, as older adults typically perform worse on these kinds of tasks compared to SM (Craik and Jennings, 1992; Spaniol et al., 2006). We found some support for this hypothesis, as there was significantly more activity for unsuccessful retrieval of personal memories in young adults, compared to older adults. We also found a marginal age difference in EM, but in this case the trend was for greater activity during non-personal episodic memories in older adults. This pattern of effects suggests that the age difference is not related to difficulty of retrieval per se, but may be related to some other process, such as retrieval of and discarding potentially relevant details. For example, activity in the SLN and cognitive-control areas during AM failures may reflect an attempt to sort through various personal details potentially relevant to the current cue, a process that would be required for AM. If this were the case, then greater activity in younger adults would be consistent with findings that younger adults typically retrieve more episodic details during AM than older adults do (Levine et al., 2002). On the other hand, older adults may retrieve an equivalent number of details when attempting to recall a non-personal episodic or semantic memory, accounting for preserved activity during EM and SM incorrect trials, which also would be consistent with their preserved or even superior semantic memory relative to younger adults (Lovden et al., 2004; Mitchell, 1989; Park et al., 2002).

We note that the effects of cognitive processing vs. RT effects cannot be disentangled in our study because taking time to search for a memory and ultimately failing to find one, or the right one, are inextricably linked. However, others have shown similar engagement of the SLN for errors in tasks where RT on error trials is actually faster than correct trials (Ham et al., 2013a). Also, since there were no age differences in RT, or a significant influence of RT on activity during memory failures in this study, RT cannot explain the age-related differences we observed in SLN activity or functional connectivity. Such findings, taken 
together with our results, provide evidence that SLN activity during errors is not strictly tied to RT.

\subsection{Functional Connectivity}

Strong functional coupling was seen within SLN regions during the incorrect trials and when the task effect was removed from the data. These connectivity patterns were very similar to each other, indicating that the SLN as a whole is involved in recall failures and that its functional connectivity is robust across a variety of cognitive states. It also is interesting that the aIFO seeds were functionally connected to cognitive-control regions, such as frontal and parietal areas thought to be nodes of the frontoparietal control network, as well as with other SLN regions. Prior work focusing on either the SLN or the FPC have identified aIFO as a participant in both of these networks (Campbell et al., 2012; Spreng et al., 2013; Vincent et al., 2008), indicating that aIFO activity may reflect the engagement of either of these two networks.

In addition to the main patterns of functional connectivity within the SLN, we also found that the aIFO seeds were functionally coupled with other regions during the retrieval conditions, and these patterns of connectivity were specific to the type of memory content that participants were attempting to retrieve, but only in the young adults. In the young group, the aIFO seeds were functionally connected to ventromedial PFC and a region in the dorsal superior frontal gyrus during AM errors. These two PFC regions are part of the default network (Grigg and Grady, 2010b; Spreng et al., 2013), which is active during AM retrieval (Addis et al., 2009; Burianova and Grady, 2007; Spreng and Grady, 2010). This pattern of connectivity may reflect the self processing that is necessary for AM retrieval, even when a memory cannot be successfully retrieved, as medial PFC is thought to be critically involved in self reference (Craik et al., 1999; Northoff and Bermpohl, 2004). In contrast, during EM and SM trials, the aIFO seeds were functionally connected with premotor and temporal areas. It is not clear what type of mechanism this pattern reflects, but it may indicate that different search processes are engaged when participants struggle to retrieve a non-personal memory.

We found no evidence for age differences in the strength of functional connectivity within the core SLN, either during the incorrect trials or in the estimate of intrinsic functional connectivity, although a number of studies have reported such age differences (Allen et al., 2011; Geerligs et al., in press; He et al., 2014; Meier et al., 2012; Onoda et al., 2012; Tomasi and Volkow, 2012). Although we did not find age differences in the primary pattern of connectivity within the SLN, we did see reductions in the strength of connectivity between the aIFO seeds and regions outside the SLN, as well as a lack of task-specificity in the pattern of connectivity across the three memory conditions in older adults. This result is consistent with the idea that brain activity is dedifferentiated or less selective in older adults (Carp et al., 2011; Grady, 2002; Park et al., 2004). Young adults showed an SLN interaction with some DMN regions that was specific to failed AM trials, which may reflect their attempt to sort through more competing details at recall, as we suggested above. This taskdependent pattern of SLN functional coupling in young adults, together with their greater activity in AM trials compared to older adults, suggests a more flexible use of the SLN 
during the engagement of AM-specific processes than in older adults, who did not show this specificity of functional connectivity.

The age difference seen in the intrinsic functional connectivity analysis consisted of weaker connectivity between aIFO and dorsomedial and lateral PFC. This age effect suggests that although older adults do not have reduced functional coupling within the SLN as a whole, they do have weaker inter-network functional connections between the SLN and the FPC (Geerligs et al., in press; He et al., 2013; He et al., 2014), as dorsolateral PFC is generally considered to be part of the FPC (Spreng et al., 2013; Vincent et al., 2008). Thus, although we found the functional connections within the SLN to be relatively maintained with age, the age differences in connections between aIFO and FPC regions, together with the dedifferentiation of connectivity patterns across retrieval conditions, suggest less flexible use of the SLN when older adults struggle to retrieve a memory. That is, if the FPC acts as a "switch" to facilitate the engagement of other brain networks, as some have suggested (Cole et al., 2013; Spreng et al., 2013), then the reduced coupling between FPC and SLN regions may indicate a reduction in the ability to flexibly "turn on" the SLN that negatively impacts its role in some types of recall in older adults.

\subsection{Conclusion}

In this experiment, we observed more activity in a distributed set of regions, consistent with the SLN, in both younger and older adults when they attempted but failed to retrieve a memory based on an external cue, compared to trials in which they successfully retrieved a memory. This activity was more prominent in younger than older adults when they attempted to retrieve a personal memory, but did not differ between groups when retrieval of EMs or SMs was unsuccessful. In addition, the functional connectivity of the SLN during unsuccessful memory retrieval was less differentiated across the memory conditions in older adults during AM and SM. These results suggest that the influence of age on brain activity during failed recall depends on the type of memory content that participants are asked to retrieve. They also support the idea that engagement of the SLN is enhanced when recall errors are made, as is the case when non-memory errors are committed. Both younger and older adults engage the SLN when recall fails, but weaker and less differentiated SLN functional connections in the older group may reduce the network's effectiveness in providing an error signal, or evaluating behavior after the error occurs, and may thus underlie some of the age differences seen in memory.

\subsection{Experimental Procedures}

\subsection{Participants and Tasks}

Fifteen young (age range $=20-33$ years, mean $=25.6, \mathrm{SD}=5.1,6$ males) and fifteen older adults (age range $=63-77$ years, mean $=69.8, \mathrm{SD}=4.7,6$ males) were recruited for this study. All participants were right-handed and native or fluent English speakers with normal or corrected-to-normal vision. Exclusion criteria included poor health conditions (e.g., back problems), history of neurological or psychiatric disorders, head injury, and stroke. The older adults all scored within the normal range (range $=27-30$, mean $=29.31$ ) on the MiniMental State Examination (Folstein et al., 1975). Informed consent was obtained in 
accordance with a protocol approved by Baycrest's Research Ethics Board. We previously reported data on task-related activity for correct responses in these participants (St-Laurent et al., 2011).

Immediately before scanning, participants received a practice session during which they were exposed to examples of the four conditions: Control, Autobiographical Memory (AM), Episodic Memory (EM), and Semantic Memory (SM). The study consisted of six fMRI runs of 498 seconds each, with 28 trials per run ( 7 trials/condition); conditions were randomized within each run. For each trial, a stimulus image was presented (4s), followed by an interstimulus interval (1s), a question (10s), and an inter-trial interval (1s). The stimuli and paradigm used here are described in greater detail in Burianova and Grady (2007). For the control trials, participants were shown a scrambled meaningless picture, and were requested to press one of three response pad keys corresponding to a letter; this task was not used in the current analysis.

For AM, EM, and SM trials, a photograph was presented with a cue word directing attention to the gist of the image (e.g., "poverty," "grandparents," "airplane"). The picture was followed by one of three types of questions (EM, AM, or SM), and participants only became aware of the condition at question onset. EM questions were about an element from the picture (e.g., "On the picture which you just saw, what was the color of the bicycle?"). SM questions were about general knowledge related to the theme of the picture (e.g., "In which city was John F. Kennedy assassinated?"). For both of these conditions, three answer choices were presented, with either button 1 or 2 corresponding to the correct answer, and button 3 corresponding to the answer "I don't know." For the current analysis, EM and SM trials were considered "correct" when participants chose the correct answer, and "incorrect" when they either selected the wrong answer or answered "I don't know". During AM trials, participants were instructed to retrieve a personal event thematically related to the picture (e.g., "Think of a time you were with older relatives"), and to rate the vividness of their memory for that event (e.g., 1 = "very vivid," 2 = "somewhat vivid," 3 = "not vivid at all"). For the current analysis, "correct" AM trials were those given "very vivid" responses, and "incorrect" AM trials were those given "somewhat vivid" or "not vivid at all" responses. Each stimulus image was presented three times over the course of the study (once in each of the three memory conditions), but never more than once per run. Accuracy was stressed over speed.

\subsection{MRI and fMRI data acquisition}

Brain images were obtained with a Siemens 3T Trio Scanner using a Matrix 12-channel head coil. The anatomical images were acquired with a T1-weighted 3D MP-RAGE oblique axial sequence (160 slices, $1 \mathrm{~mm}$ thick, FOV $=256 \mathrm{~mm}$ ). Brain activity was measured using the blood oxygenation level-dependent (BOLD) response. Functional images were acquired with an EPI oblique axial sequence $(\mathrm{TR}=2000 \mathrm{~ms}, \mathrm{TE}=30 \mathrm{~ms}, \mathrm{FOV}=200 \mathrm{~mm}$, Flip $=70$, 28 images, $5 \mathrm{~mm}$ thick).

Stimuli were projected onto a screen located behind the participant made visible through a mirror mounted on top of the head coil. Plastic goggles with corrective lenses were used when needed. Responses were made with the right hand using the first three buttons of a 
four-button Fiber-Optic Response Pad System (Current Designs Inc.). Heart rate and respiration data were collected.

Images were reconstructed and preprocessed utilizing the Analysis of Functional Neuroimages (AFNI, Cox, 1996) and Statistical Parametric Mapping (SPM5). The images were corrected for motion associated with heart rate and respiration, for the timing of the interleaved functional sequence (slice-timing), and for within-run head motion (coregistration). Images were normalized to standard MNI space using SPM5's functional EPI template and smoothed with a 6-mm Gaussian filter.

\section{3 fMRI Data Analysis}

Image analysis was conducted with partial least squares, or PLS (Krishnan et al., 2011; McIntosh et al., 1996; McIntosh and Lobaugh, 2004), a multivariate approach that identifies spatiotemporal patterns of brain activity related to tasks (task-PLS) or correlated with activity in a given brain area to assess functional connectivity (seed-PLS). PLS performs event-based signal normalization (i.e., normalizing the time points in each event to the first time point in the event), averages over all trials within a condition for each subject, and then uses singular value decomposition to extract patterns of activity that characterize the covariance between activity in all voxels and the experimental conditions or seed activity. The analysis window consisted of 8 time points $(16 \mathrm{sec})$ after the onset of the memory question. In task-PLS, each component resulting from the decomposition, known as a latent variable (LV), identifies a contrast across the experimental conditions as well as a spatial activity pattern depicting the brain regions that, as a whole, show the strongest relation to (e.g., are covariant with) the task contrast. In seed-PLS, the mean signal in a reference region is correlated with activity in all other brain voxels (within conditions, across participants) to assess the seed's functional connectivity (McIntosh, 1999). In a seed analysis, the LVs indicate the patterns of correlation, or connectivity with the seed that characterize each condition. Note that seed-PLS assesses functional connectivity across subjects, rather than calculating correlations across voxel time courses within subjects, but results in patterns of connectivity that are quite similar to those identified using withinsubject approaches (Grigg and Grady, 2010a; Grigg and Grady, 2010b). In the analyses reported here, our use of PLS was data-driven, so that each LV was extracted according to the amount of covariance in brain activity that it accounted for, similar to a principal component analysis.

Each LV has a "singular value" that indicates the amount of covariance accounted for by the LV. The significance for each LV, based on its singular value, was determined using a permutation test, with 500 permutations. These permutations use sampling without replacement to reassign the order of conditions for each participant. A PLS analysis was run for each new sample, and the number of times the permuted singular value exceeded the observed singular value was calculated for each LV. This procedure provided exact probabilities for all LVs, and an objective means for determining the number of LVs to be retained ( $p<0.05$ was used here). Because the decomposition of the data matrix is done in a single analytic step, no correction for multiple comparisons is required for this approach (McIntosh and Lobaugh, 2004). 
In addition, each brain voxel has a weight on each $\mathrm{LV}$, which is proportional to the covariance of its activity with the contrast across conditions identified by each LV. To determine which brain voxels robustly contributed to the brain pattern identified by each LV, the weights from each voxel were submitted to a bootstrap estimation (100 bootstraps) of the standard errors (SE, Efron, 1981). For the bootstrap procedure, participants were randomly resampled, with replacement, 100 times and each voxel's $S E$ was calculated. In the task PLS, all voxels where the ratio of the weight to the $S E$ for that voxel (bootstrap ratio, or BSR) exceeded \pm 4.0 were considered to make a robust contribution to the pattern. These ratios are analogous to $\mathrm{Z}$ scores (Sampson et al., 1989), so that a BSR of 4 would be equivalent to $p<0.001$. Using this BSR threshold, clusters of activity were identified that contained at least 10 voxels, and the local maximum for each cluster was defined as the voxel with a weight/SE ratio higher than any other voxel in a 2-cm cube centered on that voxel. Locations of these maxima are reported as coordinates in MNI space. To obtain summary measures of each participant's expression of each LV pattern, we calculated 'brain scores' by multiplying each voxel's weight on the LV by the BOLD signal in the voxel, and summing over all brain voxels for each participant. This resulted in a brain score for each participant in each condition, for each LV, at each time point in the analysis window. To determine task-related changes in activity, we used these "temporal brain scores", which can be thought of as hemodynamic responses of the whole-brain pattern, to assess changes across conditions and groups using repeated measures ANOVAs. For seed PLS, correlations between brain scores and seed activity were computed for each group and condition to provide a measure of how strongly the external variable covaried with the whole-brain pattern of activity. The $95 \%$ confidence intervals of the correlations were calculated via the bootstrap.

We used two kinds of analysis to examine activity related to retrieval errors. First, we used task-PLS to examine patterns of brain activity for incorrect and correct trials across the three memory tasks in younger and older adults. Both age groups were included in the analysis so that direct group differences could be assessed. The average number of correct trials included in the analysis for young adults was 23.6, 29.1 and 22.5 for AM, EM and SM, respectively; for older adults there were $23.4,27.7$ and 24.5 correct trials on average. The mean number of incorrect trials included in the analysis for young adults was 15.3, 12.1 and 16.7 for AM, EM and SM, respectively; for older adults there were 14.9, 13.7 and 13.9 incorrect trials on average.

In the seed PLS analyses, we examined functional connectivity of two important errorrelated regions during task and during an estimate of "intrinsic" or resting connectivity. These analyses were done to examine whether the regions active for incorrect responses also showed correlated activity, i.e., could be considered an integrated network of regions, and whether there were any age differences in functional connectivity. As seeds we chose the right and left aIFO as representative nodes of the SLN (Onoda et al., 2012; Seeley et al., 2007), and because these regions were more active for incorrect than for correct responses (see Figure 2). For these analyses, both young and older groups were entered, so that age differences could be assessed. 
For the analysis of task-related functional connectivity we extracted mean activity values for the two seeds from incorrect trials during the three memory conditions. To obtain representative coordinates for the seeds, we averaged the coordinates for each seed from lags 5-8 from the task PLS, as these were the lags where the BSRs were robust (resulting coordinates were $\pm 32,24,-8$ ). Then, to obtain a single estimate of activity across the trial for each seed, we extracted the mean time course for the seeds from the three conditions, and summed activity across lags 2-8 for each condition/subject, resulting in a single value per condition and subject, which were then entered into the analysis. Correlations were computed across participants between activity in each seed and all brain voxels across the conditions (resulting in 6 correlations in total for each age group). Because correlations can be driven by outliers, one younger adult and one older adult with outlying brain scores were omitted from this analysis. These outliers had extreme seed and brain score values (> 2 SDs from the mean) in two of the three memory conditions. Correlation differences between conditions and groups were determined via non-overlapping CIs.

To obtain an estimate of intrinsic functional connectivity we needed to examine correlations between error-related regions and the rest of the brain independently of task effects. This is typically done by measuring functional connectivity during resting state scans (e.g., Allen et al., 2011; Fox et al., 2005). However, since we did not obtain such scans on our participants, we took another approach, which was to treat the first run for all participants as if it were a resting state run. To do this, we divided the run into 40 sequential "blocks" of data, each 10 $\mathrm{sec}$ in length (after omitting the first $20 \mathrm{sec}$ in the run, to allow for signal equilibration), which ignores the timing of stimulus presentation, thus blurring any task effects present in the data. As with the task-related connectivity analysis, the mean signal for each seed voxel from the 40 "blocks" in the first scan was extracted and correlated with the mean signal in all other brain voxels, across participants in each group. We compared the distribution of the correlations across the 40 blocks between groups using a mixed ANOVA with group as the independent factor and hemisphere as the repeated factor. The older participant who was shown to be an outlier in the seed PLS analysis of the error trials was also omitted from this intrinsic functional connectivity analysis due to multiple outlying seed values and brain scores.

\section{Supplementary Material}

Refer to Web version on PubMed Central for supplementary material.

\section{Acknowledgments}

The authors would like to thank the Baycrest MRI technologists for technical assistance. This work was supported by the Canadian Institutes of Health Research (MOP14036), the Canada Research Chairs program, the Ontario Research Fund, the Canadian Foundation for Innovation, and the Heart and Stroke Foundation Centre for Stroke Recovery. The authors also would like to thank the following people for their generosity in support of the imaging centre at Baycrest: Jack \& Anne Weinbaum, Sam \& Ida Ross, Joseph \& Sandra Rotman.

\section{References}

Addis DR, McAndrews MP. Prefrontal and hippocampal contributions to the generation and binding of semantic associations during successful encoding. NeuroImage. 2006; 33(4):1194-206. [PubMed: 17023179] 
Addis DR, Wong AT, Schacter DL. Age-related changes in the episodic simulation of future events. Psychol Sci. 2008; 19(1):33-41. [PubMed: 18181789]

Addis DR, Pan L, Vu MA, Laiser N, Schacter DL. Constructive episodic simulation of the future and the past: distinct subsystems of a core brain network mediate imagining and remembering. Neuropsychologia. 2009; 47(11):2222-38. [PubMed: 19041331]

Addis DR, Roberts RP, Schacter DL. Age-related neural changes in autobiographical remembering and imagining. Neuropsychologia. 2011; 49(13):3656-69. [PubMed: 21945808]

Al-Aidroos N, Said CP, Turk-Browne NB. Top-down attention switches coupling between low-level and high-level areas of human visual cortex. Proc Natl Acad Sci U S A. 2012; 109(36):14675-80. [PubMed: 22908274]

Allen EA, Erhardt EB, Damaraju E, Gruner W, Segall JM, Silva RF, Havlicek M, Rachakonda S, Fries J, Kalyanam R, Michael AM, Caprihan A, Turner JA, Eichele T, Adelsheim S, Bryan AD, Bustillo J, Clark VP, Feldstein Ewing SW, Filbey F, Ford CC, Hutchison K, Jung RE, Kiehl KA, Kodituwakku P, Komesu YM, Mayer AR, Pearlson GD, Phillips JP, Sadek JR, Stevens M, Teuscher U, Thoma RJ, Calhoun VD. A baseline for the multivariate comparison of resting-state networks. Front Syst Neurosci. 2011; 5:2. [PubMed: 21442040]

Andrews-Hanna JR, Snyder AZ, Vincent JL, Lustig C, Head D, Raichle ME, Buckner RL. Disruption of large-scale brain systems in advanced aging. Neuron. 2007; 56(5):924-35. [PubMed: 18054866]

Band GP, Kok A. Age effects on response monitoring in a mental-rotation task. Biol Psychol. 2000; 51(2-3):201-21. [PubMed: 10686366]

Boly M, Phillips C, Tshibanda L, Vanhaudenhuyse A, Schabus M, Dang-Vu TT, Moonen G, Hustinx $\mathrm{R}$, Maquet $\mathrm{P}$, Laureys $\mathrm{S}$. Intrinsic brain activity in altered states of consciousness: how conscious is the default mode of brain function? Ann N Y Acad Sci. 2008; 1129:119-29. [PubMed: 18591474]

Brewer JB, Zhao Z, Desmond JE, Glover GH, Gabrieli JDE. Making memories: brain activity that predicts how well visual experience will be remembered. Science. 1998; 281:1185-1187. [PubMed: 9712581]

Burianova H, Grady CL. Common and unique neural activations in autobiographical, episodic, and semantic retrieval. J Cogn Neurosci. 2007; 19:1520-1534. [PubMed: 17714013]

Cabeza R, Nyberg L. Imaging cognition II: An empirical review of 275 PET and fMRI studies. J Cogn Neurosci. 2000; 12(1):1-47.

Cabeza R, Rao SM, Wagner AD, Mayer AR, Schacter DL. Can medial temporal lobe regions distinguish true from false? An event-related functional MRI study of veridical and illusory recognition memory. Proc Natl Acad Sci U S A. 2001; 98(8):4805-10. [PubMed: 11287664]

Cabeza, R.Nyberg, L., Park, D., editors. Cognitive Neuroscience of Aging. Oxford University Press; Oxford: 2005.

Campbell KL, Grady CL, Ng C, Hasher L. Age differences in the frontoparietal cognitive control network: Implications for distractibility. Neuropsychologia. 2012; 50(9):2212-23. [PubMed: 22659108]

Cansino S, Trejo-Morales P, Hernandez-Ramos E. Age-related changes in neural activity during source memory encoding in young, middle-aged and elderly adults. Neuropsychologia. 2010; 48(9): 2537-49. [PubMed: 20441775]

Carp J, Park J, Polk TA, Park DC. Age differences in neural distinctiveness revealed by multi-voxel pattern analysis. NeuroImage. 2011; 56:736-743. [PubMed: 20451629]

Carter CS, Braver TS, Barch DM, Botvinick MM, Noll D, Cohen JD. Anterior cingulate cortex, error detection, and the online monitoring of performance. Science. 1998; 280(5364):747-9. [PubMed: 9563953]

Cole MW, Reynolds JR, Power JD, Repovs G, Anticevic A, Braver TS. Multi-task connectivity reveals flexible hubs for adaptive task control. Nat Neurosci. 2013; 16(9):1348-55. [PubMed: 23892552]

Cox RW. AFNI: software for analysis and visualization of functional magnetic resonance neuroimages. Comput Biomed Res. 1996; 29(3):162-173. [PubMed: 8812068]

Craik, FIM., Jennings, JM. Human memory. In: Craik, FIM., Salthouse, TA., editors. The Handbook of Aging and Cognition. Lawrence Erlbaum; Hillsdale, N.J: 1992. p. 51-110.

Craik FIM, Moroz TM, Moscovitch M, Stuss DT, Winocur G, Tulving E, Kapur S. In search of the self: A positron emission tomography investigation. Psychol Sci. 1999; 10:26-34. 
Damoiseaux JS, Beckmann CF, Sanz Arigita EJ, Barkhof F, Scheltens P, Stam CJ, Smith SM, Rombouts SA. Reduced resting-state brain activity in the "default network" in normal aging. Cereb Cortex. 2008; 18:1856-1864. [PubMed: 18063564]

Dang LC, O’Neil JP, Jagust WJ. Genetic effects on behavior are mediated by neurotransmitters and large-scale neural networks. Neuroimage. 2013; 66:203-214. [PubMed: 23142068]

Daselaar SM, Fleck MS, Dobbins IG, Madden DJ, Cabeza R. Effects of Healthy Aging on Hippocampal and Rhinal Memory Functions: An Event-Related fMRI Study. Cereb Cortex. 2006; 16:1771-1782. [PubMed: 16421332]

de Chastelaine M, Wang TH, Minton B, Muftuler LT, Rugg MD. The effects of age, memory performance, and callosal integrity on the neural correlates of successful associative encoding. Cereb Cortex. 2011; 21(9):2166-76. [PubMed: 21282317]

Dennis NA, Daselaar S, Cabeza R. Effects of aging on transient and sustained successful memory encoding activity. Neurobiol Aging. 2007; 28:1749-1758. [PubMed: 16919850]

Dennis NA, Kim H, Cabeza R. Age-related differences in brain activity during true and false memory retrieval. J Cogn Neurosci. 2008; 20(8):1390-402. [PubMed: 18303982]

Donaldson DI, Wheeler ME, Petersen SE. Remember the source: dissociating frontal and parietal contributions to episodic memory. J Cogn Neurosci. 2009; 22(2):377-91.

Dosenbach NU, Visscher KM, Palmer ED, Miezin FM, Wenger KK, Kang HC, Burgund ED, Grimes AL, Schlaggar BL, Petersen SE. A core system for the implementation of task sets. Neuron. 2006; 50(5):799-812. [PubMed: 16731517]

Downar J, Crawley AP, Mikulis DJ, Davis KD. A cortical network sensitive to stimulus salience in a neutral behavioral context across multiple sensory modalities. J Neurophysiol. 2002; 87(1):61520. [PubMed: 11784775]

Duarte A, Henson RN, Graham KS. The effects of aging on the neural correlates of subjective and objective recollection. Cereb Cortex. 2008; 18(9):2169-80. [PubMed: 18165281]

Duarte A, Graham KS, Henson RN. Age-related changes in neural activity associated with familiarity, recollection and false recognition. Neurobiol Aging. 2010; 31(10):1814-30. [PubMed: 19004526]

Duverne S, Motamedinia S, Rugg MD. The relationship between aging, performance, and the neural correlates of successful memory encoding. Cereb Cortex. 2009; 19(3):733-44. [PubMed: 18653664]

Efron B. Nonparametric estimates of standard error: The jackknife, the bootstrap, and other methods. Biometrika. 1981; 68:589-599.

Endrass T, Schreiber M, Kathmann N. Speeding up older adults: age-effects on error processing in speed and accuracy conditions. Biol Psychol. 2012; 89(2):426-32. [PubMed: 22197882]

Falkenstein M, Hoormann J, Hohnsbein J. Changes of error-related ERPs with age. Exp Brain Res. 2001; 138(2):258-62. [PubMed: 11417467]

Farb NA, Grady CL, Strother S, Tang-Wai DF, Masellis M, Black S, Freedman M, Pollock BG, Campbell KL, Hasher L, Chow TW. Abnormal network connectivity in frontotemporal dementia: Evidence for prefrontal isolation. Cortex. 2013; 49(7):1856-1873. [PubMed: 23092697]

Folstein MF, Folstein SE, McHugh PR. "Mini Mental State"- a practical method for grading the cognitive state of patients for the clinician. J Psychiatr Res. 1975; 12:189-198. [PubMed: 1202204]

Fox MD, Snyder AZ, Vincent JL, Corbetta M, Van Essen DC, Raichle ME. The human brain is intrinsically organized into dynamic, anticorrelated functional networks. Proc Nat Acad Sci. 2005; 102(27):9673-8. [PubMed: 15976020]

Garoff-Eaton RJ, Slotnick SD, Schacter DL. Not all false memories are created equal: The neural basis of false recognition. Cereb Cortex. 2006; 16(11):1645-1652. [PubMed: 16400158]

Geerligs L, Renken RJ, Saliasi E, Maurits NM, Lorist MM. A Brain-Wide Study of Age-Related Changes in Functional Connectivity. Cereb Cortex. in press.

Giovanello KS, Kensinger EA, Wong AT, Schacter DL. Age-related neural changes during memory conjunction errors. J Cogn Neurosci. 2010; 22(7):1348-61. [PubMed: 19445606]

Giovanello KS, Schacter DL. Reduced specificity of hippocampal and posterior ventrolateral prefrontal activity during relational retrieval in normal aging. J Cogn Neurosci. 2012; 24(1):159-70. [PubMed: 21812566] 
Grady C. The cognitive neuroscience of ageing. Nat Rev Neurosci. 2012; 13(7):491-505. [PubMed: 22714020]

Grady CL. Age-related differences in face processing: A meta-analysis of three functional neuroimaging experiments. Canad J Exp Psychol. 2002; 56:208-220. [PubMed: 12271751]

Grady CL, Grigg O, Ng C. Age differences in default and reward networks during processing of personally relevant information. Neuropsychologia. 2012; 50(7):1682-97. [PubMed: 22484520]

Grady CL, Mondloch CJ, Lewis TL, Maurer D. Early visual deprivation from congenital cataracts disrupts activity and functional connectivity in the face network. Neuropsychologia. 2014; 57:12239. [PubMed: 24657305]

Grigg O, Grady CL. Task-related effects on the temporal and spatial dynamics of resting-state functional connectivity in the default network. PLoS ONE. 2010a; 5(10):e13311. [PubMed: 20967203]

Grigg O, Grady CL. The default network and processing of personally relevant information: Converging evidence from task-related modulations and functional connectivity. Neuropsychologia. 2010b; 48:3815-3823. [PubMed: 20837034]

Gutchess AH, Welsh RC, Hedden T, Bangert A, Minear M, Liu LL, Park DC. Aging and the neural correlates of successful picture encoding: Frontal activations compensate for decreased medial temporal activity. J Cogn Neurosci. 2005; 17:84-96. [PubMed: 15701241]

Ham T, Leff A, de Boissezon X, Joffe A, Sharp DJ. Cognitive Control and the Salience Network: An Investigation of Error Processing and Effective Connectivity. J Neurosci. 2013a; 33(16):70917098. [PubMed: 23595766]

Ham TE, de Boissezon X, Leff A, Beckmann C, Hughes E, Kinnunen KM, Leech R, Sharp DJ. Distinct frontal networks are involved in adapting to internally and externally signaled errors. Cereb Cortex. 2013b; 23(3):703-13. [PubMed: 22426336]

He X, Qin W, Liu Y, Zhang X, Duan Y, Song J, Li K, Jiang T, Yu C. Age-related decrease in functional connectivity of the right fronto-insular cortex with the central executive and default-mode networks in adults from young to middle age. Neurosci Lett. 2013; 544:74-9. [PubMed: 23583587]

He X, Qin W, Liu Y, Zhang X, Duan Y, Song J, Li K, Jiang T, Yu C. Abnormal salience network in normal aging and in amnestic mild cognitive impairment and Alzheimer's disease. Hum Brain Mapp. 2014; 35(7):3446-3464. [PubMed: 24222384]

Henson RN, Shallice T, Dolan RJ. Right prefrontal cortex and episodic memory retrieval: a functional MRI test of the monitoring hypothesis. Brain. 1999; 122:1367-1381. [PubMed: 10388802]

Holroyd CB, Coles MG. The neural basis of human error processing: reinforcement learning, dopamine, and the error-related negativity. Psychol Rev. 2002; 109(4):679-709. [PubMed: 12374324]

Kalpouzos G, Persson J, Nyberg L. Local brain atrophy accounts for functional activity differences in normal aging. Neurobiol Aging. 2012; 33(3):623 e1-623 e13.

Kelly AM, Uddin LQ, Biswal BB, Castellanos FX, Milham MP. Competition between functional brain networks mediates behavioral variability. Neuroimage. 2008; 39(1):527-37. [PubMed: 17919929]

Klein TA, Endrass T, Kathmann N, Neumann J, von Cramon DY, Ullsperger M. Neural correlates of error awareness. Neuroimage. 2007; 34(4):1774-81. [PubMed: 17185003]

Krishnan A, Williams LJ, McIntosh AR, Abdi H. Partial Least Squares (PLS) methods for neuroimaging: a tutorial and review. NeuroImage. 2011; 56(2):455-75. [PubMed: 20656037]

Levine B, Svoboda E, Hay J, Winocur G, Moscovitch M. Aging and autobiographical memory: dissociating episodic from semantic retrieval. Psychol Aging. 2002; 17:677-689. [PubMed: 12507363]

Lovden M, Ronnlund M, Wahlin A, Backman L, Nyberg L, Nilsson LG. The extent of stability and change in episodic and semantic memory in old age: demographic predictors of level and change. $\mathrm{J}$ Gerontol B Psychol Sci Soc Sci. 2004; 59(3):P130-4. [PubMed: 15118016]

Maguire EA, Frith CD. Aging affects the engagement of the hippocampus during autobiographical memory retrieval. Brain. 2003; 126(Pt 7):1511-23. [PubMed: 12805116]

Maril A, Wagner AD, Schacter DL. On the tip of the tongue: an event-related fMRI study of semantic retrieval failure and cognitive conflict. Neuron. 2001; 31(4):653-660. [PubMed: 11545723] 
Maril A, Simons JS, Weaver JJ, Schacter DL. Graded recall success: an event-related fMRI comparison of tip of the tongue and feeling of knowing. Neuroimage. 2005; 24(4):1130-8. [PubMed: 15670690]

McIntosh AR, Bookstein FL, Haxby JV, Grady CL. Spatial pattern analysis of functional brain images using Partial Least Squares. NeuroImage. 1996; 3:143-157. [PubMed: 9345485]

McIntosh AR. Mapping cognition to the brain through neural interactions. Memory. 1999; 7:523-548. [PubMed: 10659085]

McIntosh AR, Lobaugh NL. Partial least squates analysis of neuroimaging data: applications and advances. Neuroimage. 2004; 23(Supplement 1):S250-S263. [PubMed: 15501095]

Meier TB, Desphande AS, Vergun S, Nair VA, Song J, Biswal BB, Meyerand ME, Birn RM, Prabhakaran V. Support vector machine classification and characterization of age-related reorganization of functional brain networks. Neuroimage. 2012; 60(1):601-13. [PubMed: 22227886]

Mennes M, Kelly C, Colcombe S, Castellanos FX, Milham MP. The extrinsic and intrinsic functional architectures of the human brain are not equivalent. Cereb Cortex. 2013; 23(1):223-9. [PubMed: 22298730]

Mitchell DB. How many memory systems? Evidence from aging. J Exp Psychol Learn Mem Cogn. 1989; 15(1):31-49. [PubMed: 2522140]

Morcom AM, Good CD, Frackowiak RS, Rugg MD. Age effects on the neural correlates of successful memory encoding. Brain. 2003; 126(Pt 1):213-229. [PubMed: 12477708]

Morcom AM, Li J, Rugg MD. Age effects on the neural correlates of episodic retrieval: Increased cortical recruitment with matched performance. Cereb Cortex. 2007; 17:2491-2506. [PubMed: 17204820]

Moscovitch M. Memory and working-with-memory: A component process model based on modules and central systems. J Cogn Neurosci. 1992; 4:257-267. [PubMed: 23964882]

Nieuwenhuis S, Ridderinkhof KR, Talsma D, Coles MG, Holroyd CB, Kok A, van der Molen MW. A computational account of altered error processing in older age: dopamine and the error-related negativity. Cogn Affect Behav Neurosci. 2002; 2(1):19-36. [PubMed: 12452582]

Northoff G, Bermpohl F. Cortical midline structures and the self. Trends Cogn Sci. 2004; 8(3):102-7. [PubMed: 15301749]

Okado Y, Stark C. Neural processing associated with true and false memory retrieval. Cogn Affect Behav Neurosci. 2003; 3(4):323-34. [PubMed: 15040552]

Onoda K, Ishihara M, Yamaguchi S. Decreased functional connectivity by aging is associated with cognitive decline. J Cogn Neurosci. 2012; 24(11):2186-98. [PubMed: 22784277]

Park DC, Lautenschlager G, Hedden T, Davidson NS, Smith AD, Smith PK. Models of visuospatial and verbal memory across the adult life span. Psychol Aging. 2002; 17(2):299-320. [PubMed: 12061414]

Park DC, Polk TA, Park R, Minear M, Savage A, Smith MR. Aging reduces neural specialization in ventral visual cortex. Proc Natl Acad Sci U S A. 2004; 101(35):13091-5. [PubMed: 15322270]

Piefke M, Fink GR. Recollections of one's own past: the effects of aging and gender on the neural mechanisms of episodic autobiographical memory. Anat Embryol (Berl). 2005; 210(5-6):497-512. [PubMed: 16172875]

Rajah MN, D'Esposito M. Region-specific changes in prefrontal function with age: a review of PET and fMRI studies on working and episodic memory. Brain. 2005; 128(Pt 9):1964-83. [PubMed: 16049041]

Rajah MN, Kromas M, Han JE, Pruessner JC. Group differences in anterior hippocampal volume and in the retrieval of spatial and temporal context memory in healthy young versus older adults. Neuropsychologia. 2010; 48(14):4020-30. [PubMed: 20946907]

Rieckmann A, Karlsson S, Fischer H, Backman L. Caudate dopamine D1 receptor density is associated with individual differences in frontoparietal connectivity during working memory. J Neurosci. 2011; 31(40):14284-90. [PubMed: 21976513]

Rubia K, Smith AB, Taylor E, Brammer M. Linear age-correlated functional development of right inferior fronto-striato-cerebellar networks during response inhibition and anterior cingulate during error-related processes. Hum Brain Mapp. 2007; 28(11):1163-77. [PubMed: 17538951] 
Rugg MD, Otten LJ, Henson RN. The neural basis of episodic memory: evidence from functional neuroimaging. Philosophical Transactions of the Royal Society of London B: Biological Science. 2002; 357(1424):1097-1110.

Sampson PD, Streissguth AP, Barr HM, Bookstein FL. Neurobehavioral effects of prenatal alchohol: Part II. Partial least squares analysis. Neurotox Teratol. 1989; 11:477-491.

Seeley WW, Menon V, Schatzberg AF, Keller J, Glover GH, Kenna H, Reiss AL, Greicius MD. Dissociable intrinsic connectivity networks for salience processing and executive control. J Neurosci. 2007; 27(9):2349-56. [PubMed: 17329432]

Slotnick SD, Schacter DL. A sensory signature that distinguishes true from false memories. Nat Neurosci. 2004; 7(6):664-72. [PubMed: 15156146]

Smith SM, Fox PT, Miller KL, Glahn DC, Fox PM, Mackay CE, Filippini N, Watkins KE, Toro R, Laird AR, Beckmann CF. Correspondence of the brain's functional architecture during activation and rest. Proc Natl Acad Sci U S A. 2009; 106(31):13040-5. [PubMed: 19620724]

Spaniol J, Madden DJ, Voss A. A diffusion model analysis of adult age differences in episodic and semantic long-term memory retrieval. J Exp Psychol Learn Mem Cogn. 2006; 32(1):101-17. [PubMed: 16478344]

Spaniol J, Davidson PS, Kim AS, Han H, Moscovitch M, Grady CL. Event-related fMRI studies of episodic encoding and retrieval: meta-analyses using activation likelihood estimation. Neuropsychologia. 2009; 47(8-9):1765-79. [PubMed: 19428409]

Spaniol J, Grady C. Aging and the neural correlates of source memory: over-recruitment and functional reorganization. Neurobiol Aging. 2012; 33:425.e3-425.e18.

Spreng RN, Grady CL. Patterns of brain activity supporting autobiographical memory, prospection, and theory of mind, and their relationship to the default mode network. J Cogn Neurosci. 2010; 22(6):1112-23. [PubMed: 19580387]

Spreng RN, Sepulcre J, Turner GR, Stevens WD, Schacter DL. Intrinsic architecture underlying the relations among the default, dorsal attention, and frontoparietal control networks of the human brain. J Cogn Neurosci. 2013; 25(1):74-86. [PubMed: 22905821]

St Jacques PL, Levine B. Ageing and autobiographical memory for emotional and neutral events. Memory. 2007; 15(2):129-44. [PubMed: 17534107]

St-Laurent M, Abdi H, Burianova H, Grady CL. Influence of Aging on the Neural Correlates of Autobiographical, Episodic, and Semantic Memory Retrieval. J Cogn Neurosci. 2011; 23(12): 4150-4163. [PubMed: 21671743]

Stevens WD, Hasher L, Chiew K, Grady CL. A neural mechanism underlying memory failure in older adults. J Neurosci. 2008; 28:12820-12824. [PubMed: 19036975]

Thomas JB, Brier MR, Snyder AZ, Vaida FF, Ances BM. Pathways to neurodegeneration: Effects of HIV and aging on resting-state functional connectivity. Neurology. 2013; 80(13):1186-93. [PubMed: 23446675]

Tomasi D, Volkow ND. Aging and functional brain networks. Mol Psychiatry. 2012; 17(5):471, 549_ 58. [PubMed: 21727896]

van Veen V, Carter CS. Error detection, correction, and prevention in the brain: a brief review of data and theories. Clin EEG Neurosci. 2006; 37(4):330-5. [PubMed: 17073172]

Vilberg KL, Rugg MD. Memory retrieval and the parietal cortex: a review of evidence from a dualprocess perspective. Neuropsychologia. 2008; 46(7):1787-99. [PubMed: 18343462]

Vincent JL, Kahn I, Snyder AZ, Raichle ME, Buckner RL. Evidence for a frontoparietal control system revealed by intrinsic functional connectivity. J Neurophysiol. 2008; 100(6):3328-42. [PubMed: 18799601]

Voss MW, Prakash RS, Erickson KI, Basak C, Chaddock L, Kim JS, Alves H, Heo S, Szabo AN, White SM, Wojcicki TR, Mailey EL, Gothe N, Olson EA, McAuley E, Kramer AF. Plasticity of brain networks in a randomized intervention trial of exercise training in older adults. Front Aging Neurosci. 2010; 2doi: 10.3389/fnagi.2010.00032

Wagner AD, Schacter DL, Rotte M, Koutstaal W, Maril A, Dale AM, Rosen BR, Buckner RL. Building memories: remembering and forgetting of verbal experiences as predicted by brain activity. Science. 1998; 281:1188-1191. [PubMed: 9712582] 
Wang L, Su L, Shen H, Hu D. Decoding lifespan changes of the human brain using resting-state functional connectivity MRI. PLoS One. 2012; 7(8):e44530. [PubMed: 22952990]

Wheeler ME, Petersen SE, Nelson SM, Ploran EJ, Velanova K. Dissociating early and late error signals in perceptual recognition. J Cogn Neurosci. 2008; 20(12):2211-25. [PubMed: 18457507] 


\section{Highlights (for review)}

- The salience network is engaged for memory recall failures in younger and older adults.

- Older adults show weaker functional connectivity involving the salience network.

- Older adults also show dedifferentiation of salience network functional connectivity.

- Older adults may have less flexible engagement of the salience network during recall failures. 
$\square$ Young Adults $\square$ Older Adults

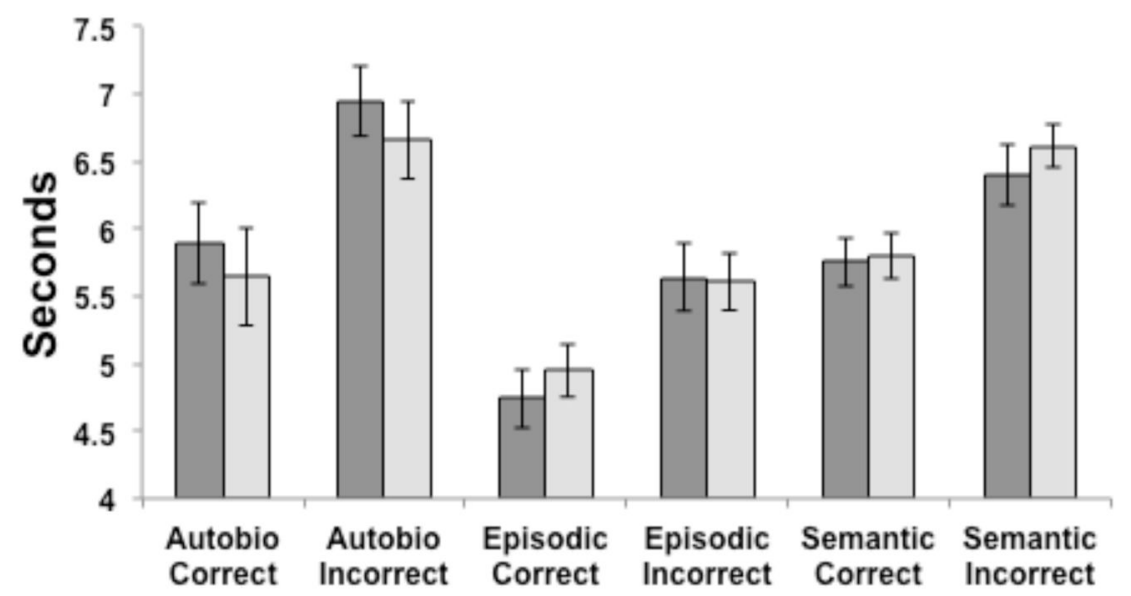

Figure 1.

Response times for correct and incorrect responses across the three memory conditions for young and older adults. Error bars represent the S.E. 


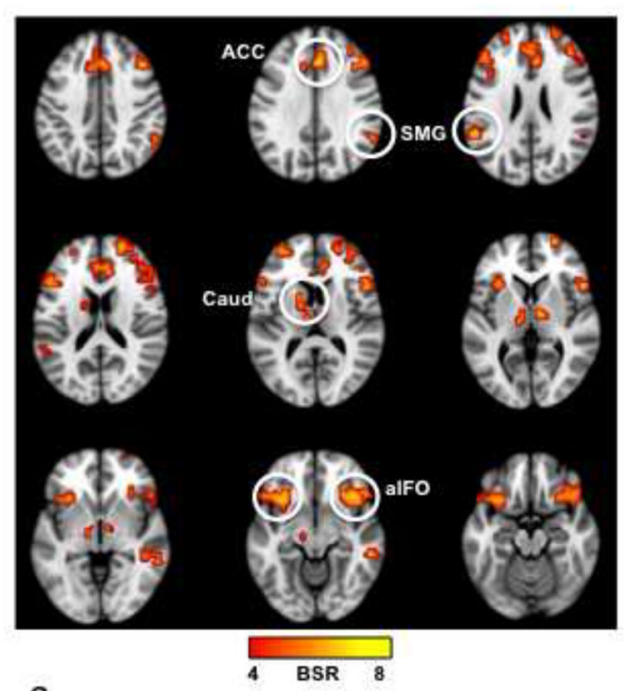

a

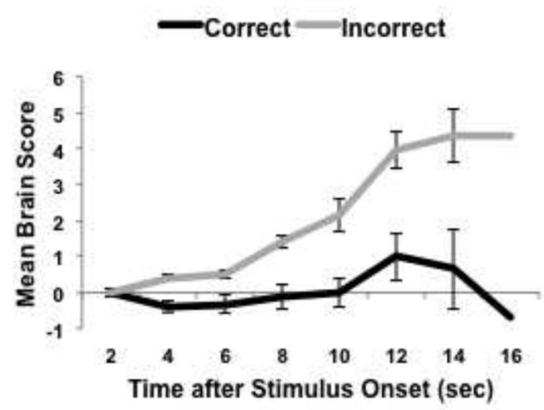

b

Figure 2.

(a) Brain areas with more activity for incorrect responses (image taken from the $8^{\text {th }}$ time point, i.e., $16 \mathrm{sec}$ after the beginning of the trial, where the difference between correct and incorrect responses was maximal). BSR = bootstrap ratio; the color bar indicates the range of BSRs used to make the brain image. Regions indicated by circles: ACC, anterior cingulate; aIFO, anterior insula/frontal operculum; Caud, caudate nucleus, SMG, supramarginal gyrus. (b) Mean brain scores collapsed across groups and condition. The brain score is a summary measure that indicates the degree to which each participant expresses the activity pattern seen in (a). If the mean brain score is positive at a given time, this indicates more activity relative to the first time point in the trial (i.e., 0-2 sec after stimulus onset). Error bars represent the S.E. 

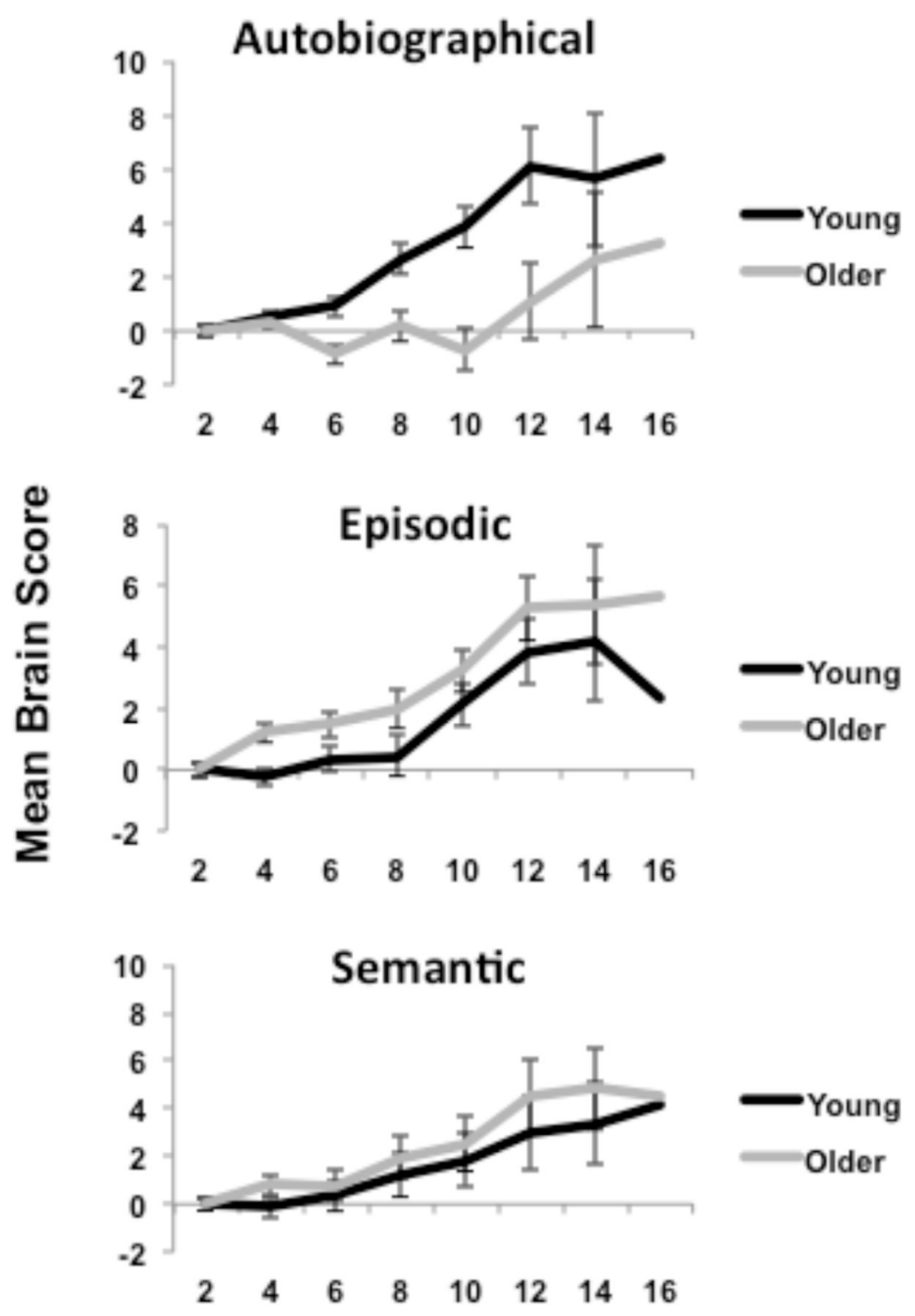

Time after Stimulus Onset (sec)

Figure 3.

Mean brain scores for incorrect trials are plotted for each group and memory condition across time. The brain score is a summary measure that indicates the degree to which each participant expresses the activity pattern seen in Figure 2a. Error bars represent the S.E. 


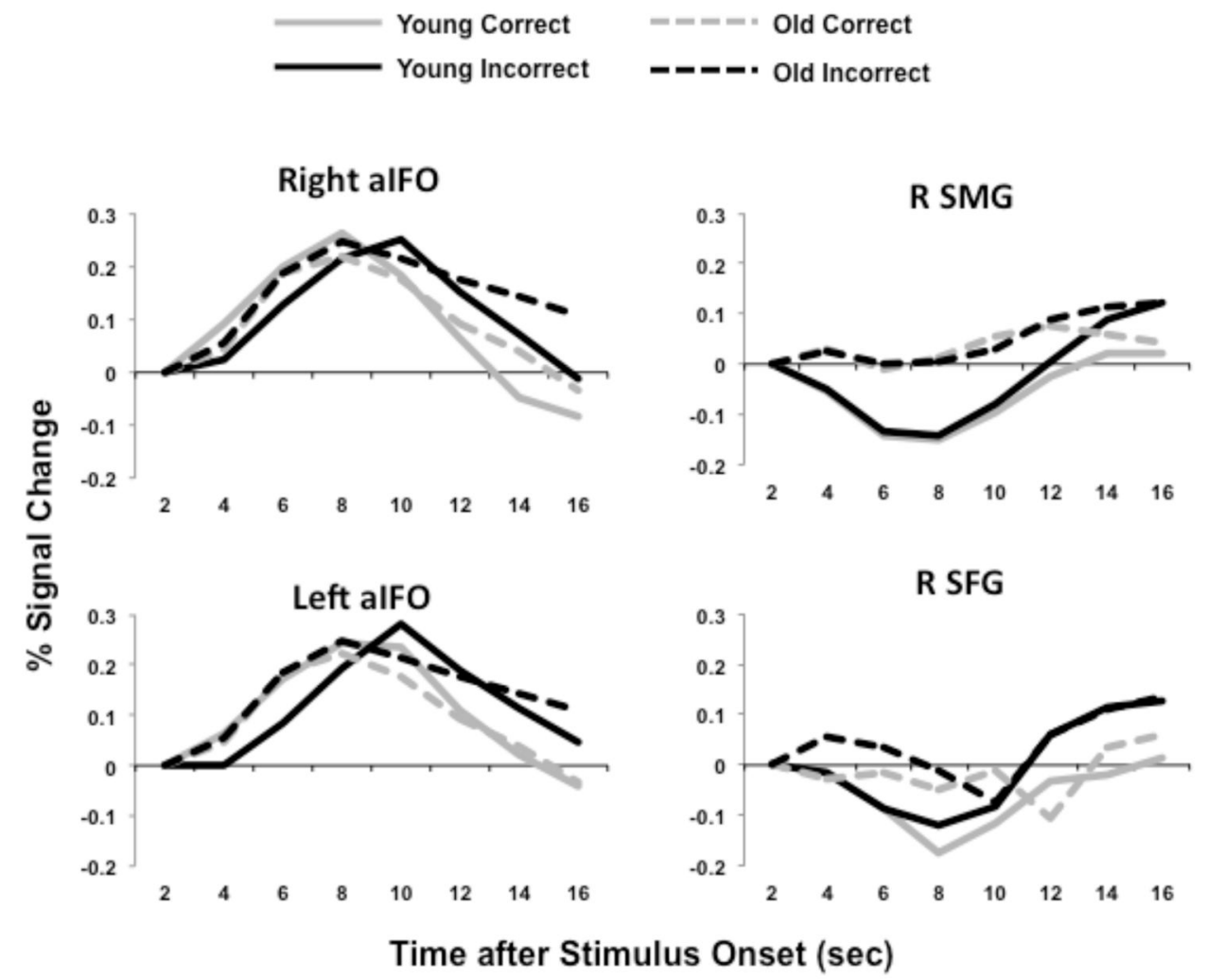

Figure 4.

The hemodynamic responses for four representative regions are plotted for younger (solid lines) and older adults (dashed lines). In both groups activity was increased in the right and left aIFO seeds during correct and incorrect trials, but either peaked later for incorrect trials, and/or was more sustained for incorrect trials. Response profiles of right supramarginal gyrus (SMG) and right superior frontal gyrus (SFG) are flatter, but still show differentiation between correct and incorrect responses late in the trial. 


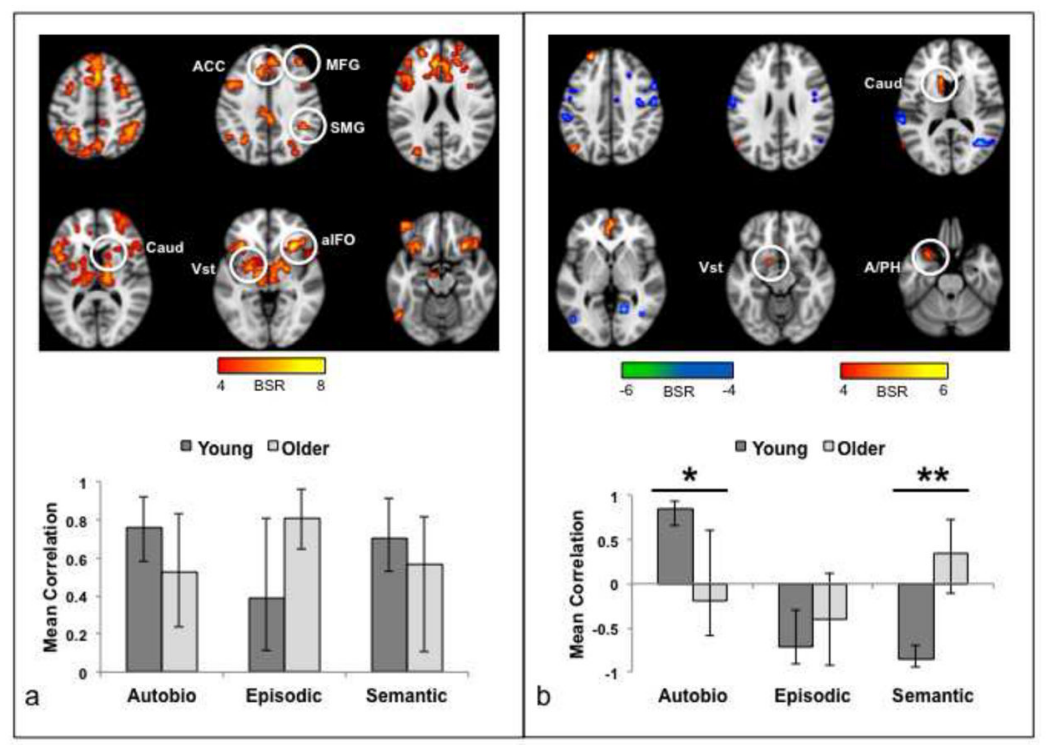

Figure 5.

Results of the functional connectivity analysis for the aIFO seeds during the incorrect trials. (a) The brain image shows the regions where activity was robustly correlated with the seeds in the first significant pattern. The graph shows the mean correlation (averaged for right and left aIFO) between seed activity and the brain scores (i.e., the summary measure of functional connectivity for each participant). The brain measures were averaged across right and left hemispheres because there were no differences in the correlations for right and left aIFO in either group. There were no age differences in the correlations or differences across memory condition. Error bars are the average confidence intervals for left and right seed correlations. (b) The brain image shows the regions where activity was robustly correlated with the seeds in the second significant pattern. The graph shows the mean correlation (averaged for right and left aIFO). Areas shown in warm colors were positively correlated with the seeds during AM in young adults and cool colored areas were positively correlated with the seeds for EM and SM in young adults. $*=\mathrm{O}<\mathrm{Y}$ for right aIFO. $* *=\mathrm{O}<\mathrm{Y}$ for both seeds (see Table 2 for the correlation values for each seed and group). Error bars are the average confidence intervals for left and right seed correlations. The color bars indicate the range of BSRs used to make the brain images. Regions indicated by circles: ACC, anterior cingulate; aIFO, anterior insula/frontal operculum; Vst, ventral striatum; Caud, caudate nucleus; SMG, supramarginal gyrus; MFG, middle frontal gyrus; A/PH, amygdala/ parahippocampal gyrus. 


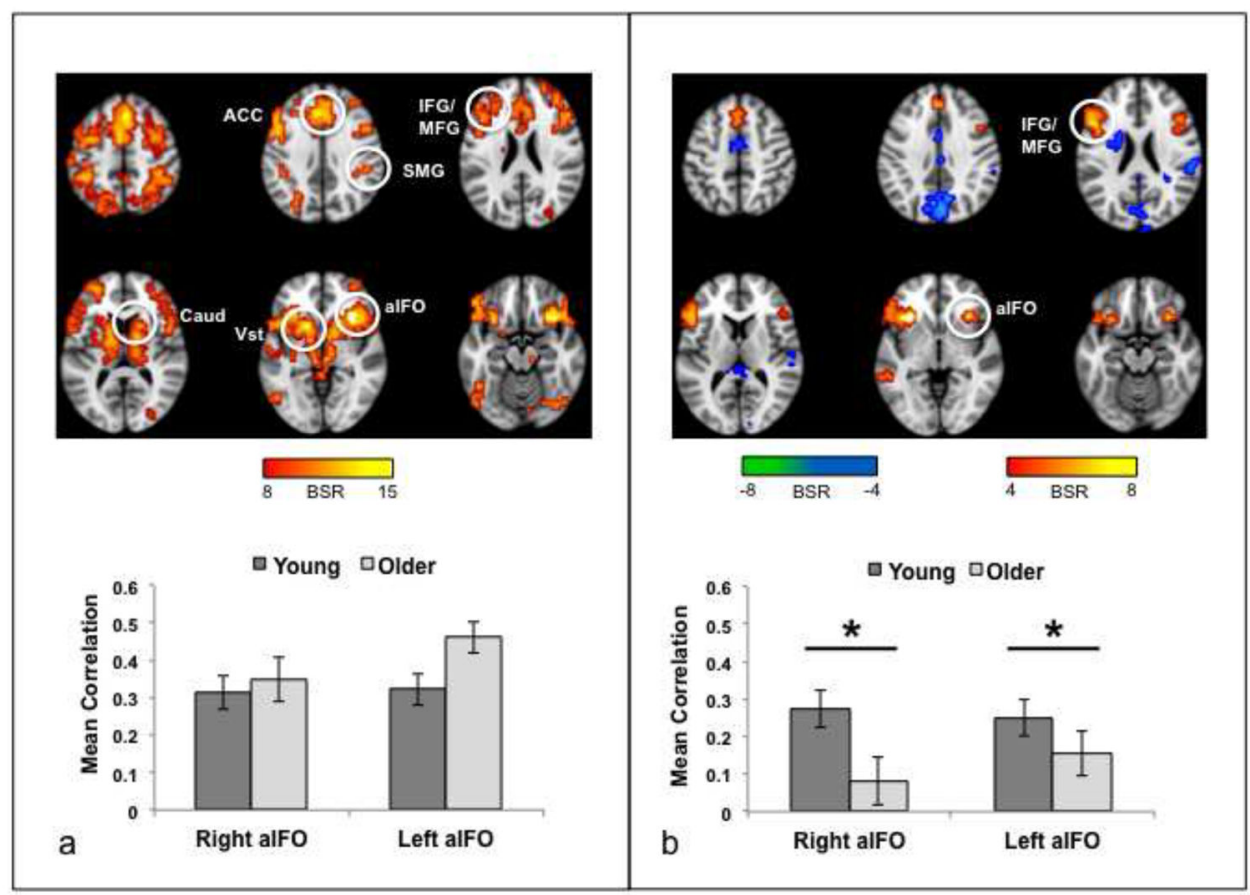

Figure 6.

Results of the intrinsic functional connectivity analysis for aIFO. (a) The brain image shows the regions where activity was robustly correlated with the seeds for the first significant pattern. The graph shows the mean correlation (averaged across the 40 "blocks" of the first scanning run) between activity in each seed and the brain scores (i.e., the summary measure of functional connectivity for each participant). There were no age differences in the correlations. (b) The brain image shows the regions where activity was robustly correlated with the seeds for the second significant pattern. Warm colored regions are positively correlated with the seeds and cool colored regions are negatively correlated with the seeds. The graph shows the mean correlation between activity in each seed and the brain scores. * $\mathrm{O}<\mathrm{Y}$ for both seeds. Error bars represent the SE of the mean. The color bars indicate the range of BSRs used to make the brain images. Regions indicated by circles: ACC, anterior cingulate; aIFO, anterior insula/frontal operculum; Vst, ventral striatum; Caud, caudate nucleus; SMG, supramarginal gyrus; IFG/MFG, inferior and middle frontal gyri. 


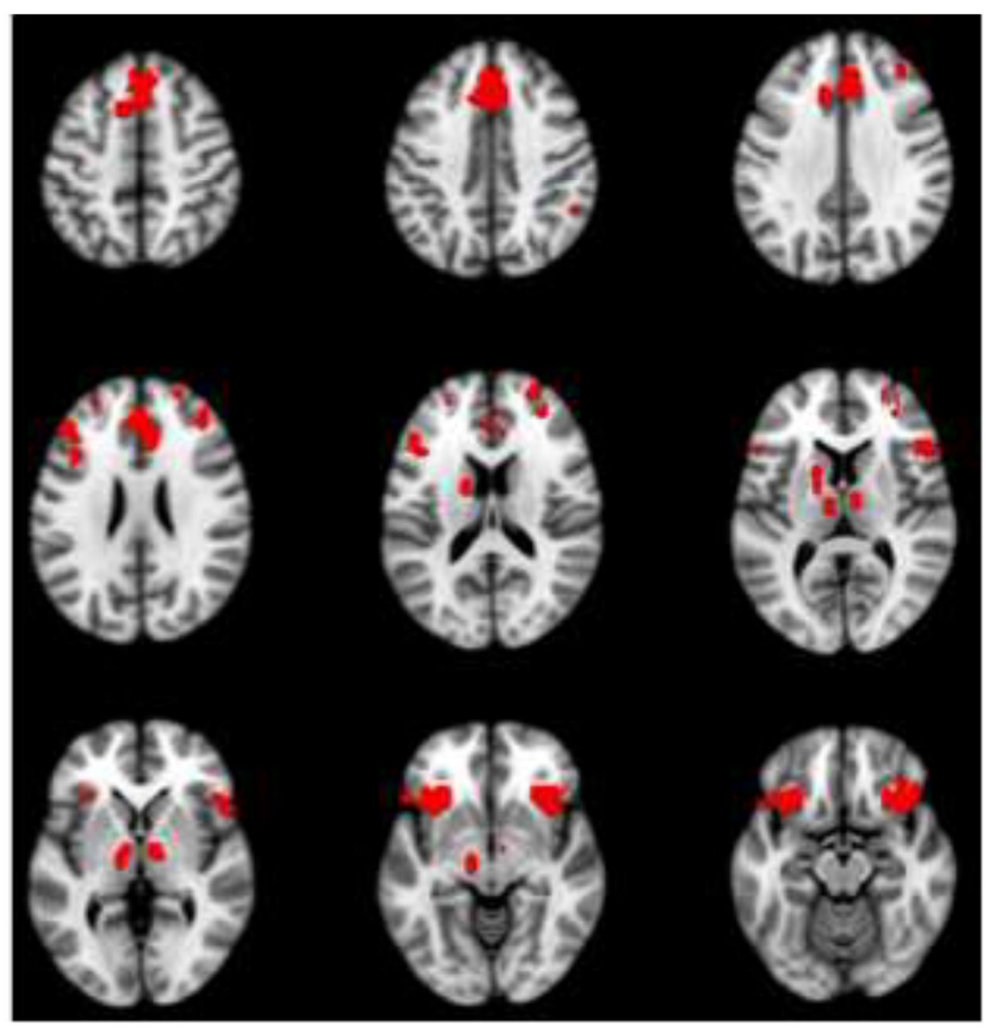

Figure 7.

The red regions indicate where there was overlap among the regions with more activity for incorrect trials (see Figure 2a), strong functional connectivity with aIFO seeds during incorrect trials (see Figure 5a) and strong intrinsic functional connectivity with aIFO seeds (see Figure 6a). These regions are consistent with the SLN, indicating that this network is engaged for recall errors and shows robust intrinsic and task-related functional connectivity. To make this overlap map, the BSR thresholds from the original images were used (see Figures 2a, 5a and 6a). 


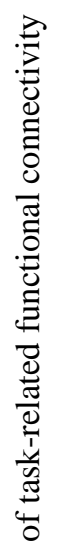




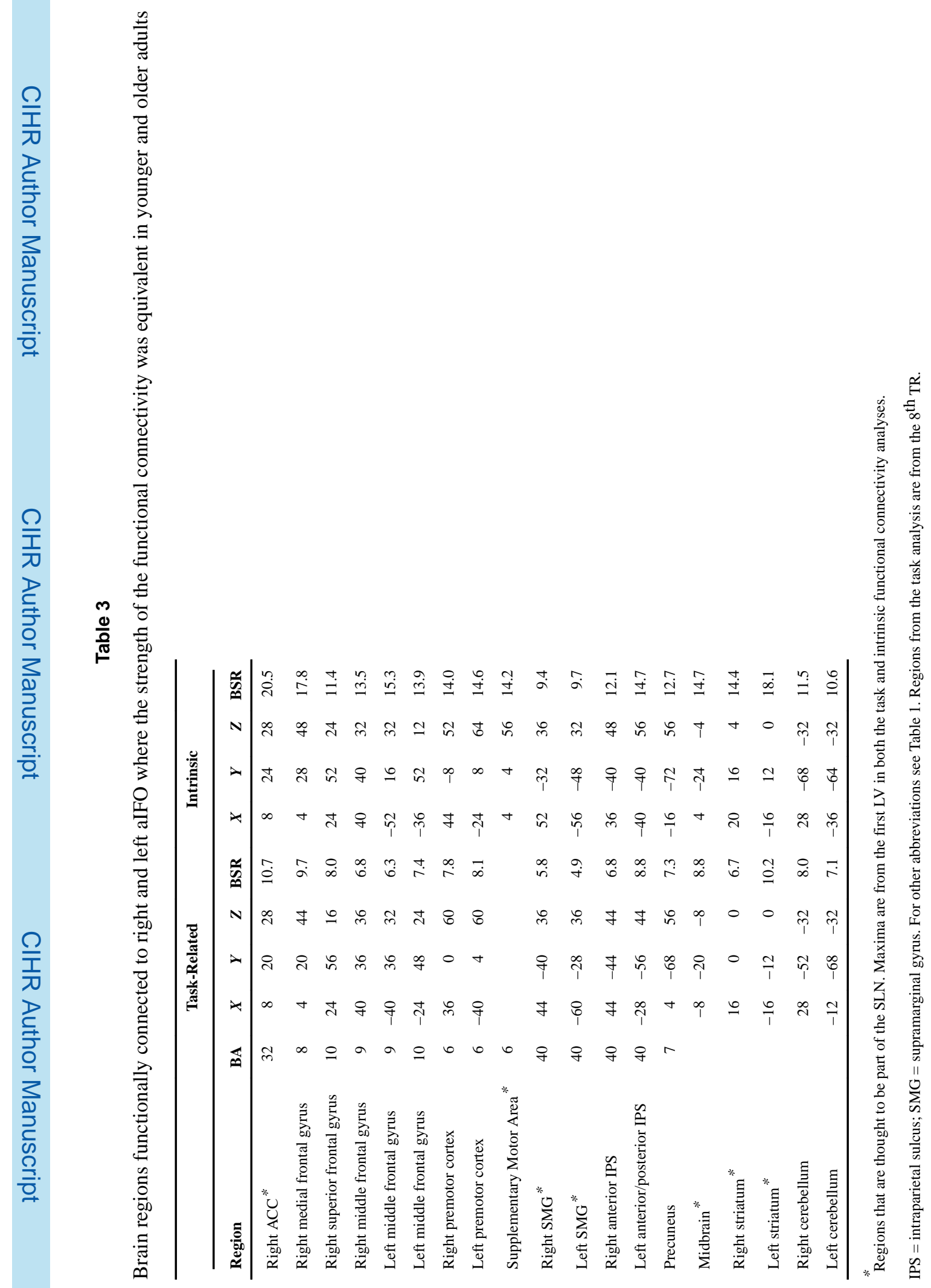




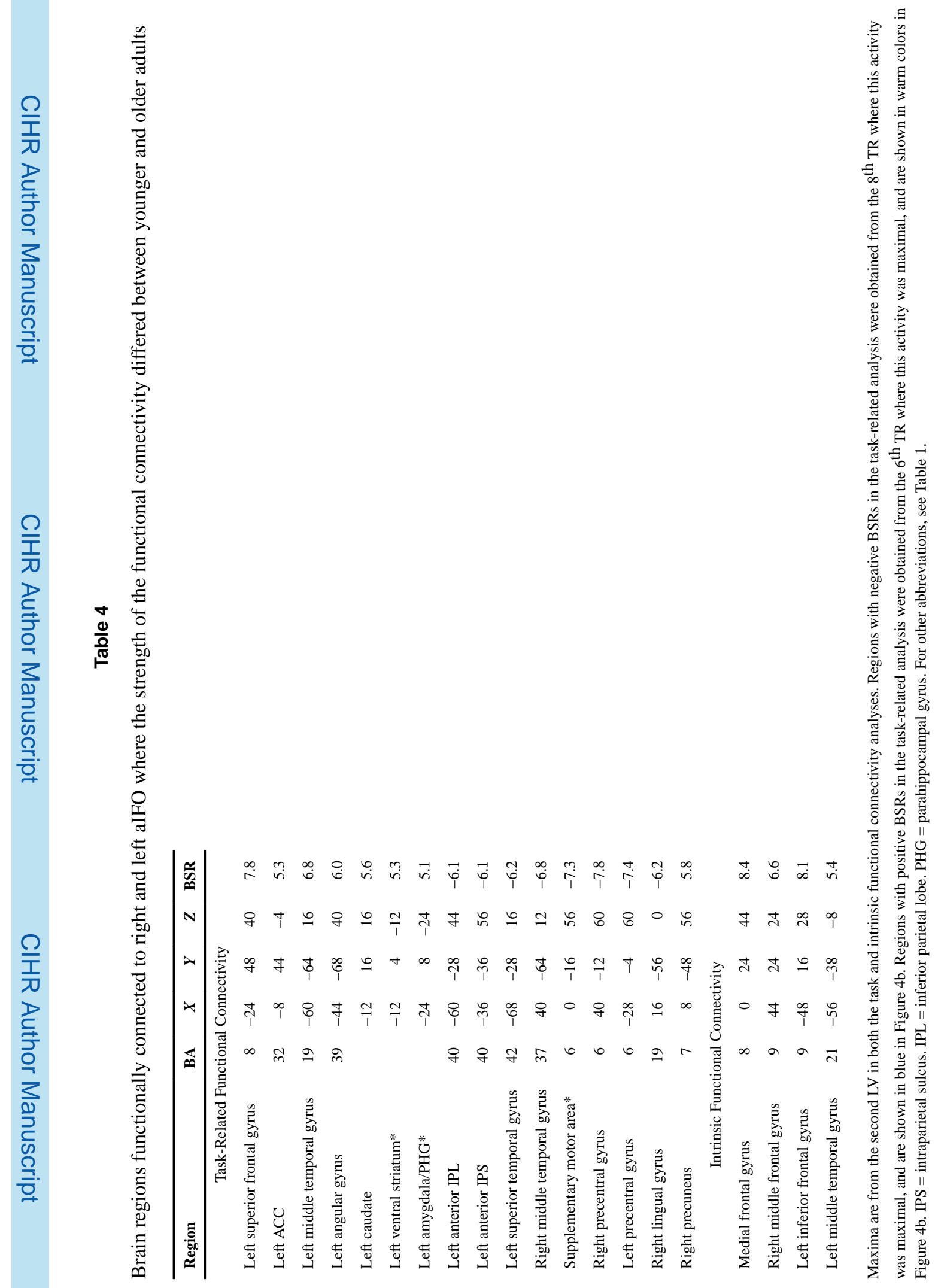

